\title{
On the local spectral properties of weighted shift operators
}

by

\section{A. Bourhim (Trieste)}

\begin{abstract}
We study the local spectral properties of both unilateral and bilateral weighted shift operators.
\end{abstract}

1. Introduction. The weighted shift operators have proved to be a very interesting collection of operators, providing examples and counter-examples to illustrate many properties of operators. The basic facts concerning their spectral theory have been fully investigated, and can be found in A. Shields' excellent survey article [20]. The description of their local spectra, and the characterization of their local spectral properties is a natural problem, and there has been some progress in this direction. In K. Laursen and M. Neumann's remarkable monograph [13], it is shown that each local spectrum of a unilateral weighted shift operator $S$ contains a closed disc centered at the origin. Sufficient conditions are given for a unilateral weighted shift operator $S$ to satisfy Dunford's condition $(C)$ and for $S$ not to have Bishop's property $(\beta)$. It is also shown that a unilateral weighted shift operator is decomposable if and only if it is quasi-nilpotent. But it has been unknown which unilateral or bilateral weighted shift operators satisfy Dunford's condition $(C)$ or have Bishop's property $(\beta)$. It has also been unknown which bilateral weighted shift operators are decomposable.

The main goal of this paper is to study the local spectral properties of both unilateral and bilateral weighted shift operators. We aim at determining the local spectra of a weighted shift $S$ in terms of the weight sequence defining $S$. Several necessary and sufficient conditions for $S$ to satisfy Dunford's condition $(C)$ or Bishop's property $(\beta)$ are given.

Throughout this paper, $\mathcal{H}$ will denote a complex Hilbert space, and $\mathcal{L}(\mathcal{H})$ the algebra of all bounded linear operators on $\mathcal{H}$. For an operator $T \in \mathcal{L}(\mathcal{H})$, let $T^{*}, \sigma(T), \sigma_{\text {ap }}(T), \sigma_{\mathrm{p}}(T), W(T), r(T)$, and $w(T)$ de-

2000 Mathematics Subject Classification: Primary 47B37; Secondary 47A10, 47A11, $47 \mathrm{~A} 12$.

The research author was supported in part by the Abdus Salam International Centre for Theoretical Physics, Trieste, Italy. 
note the adjoint, spectrum, approximate point spectrum, point spectrum, numerical range, spectral radius, and numerical radius of $T$, respectively. Let $m(T):=\inf \{\|T x\|:\|x\|=1\}$ denote the lower bound of $T$, and set $r_{1}(T):=\sup _{n \geq 1} m\left(T^{n}\right)^{1 / n}$. In [14], E. Makai and J. Zemánek showed that the sequence $\left(m\left(T^{n}\right)^{1 / n}\right)_{n \geq 1}$ converges and

$$
r_{1}(T)=\lim _{n \rightarrow \infty} m\left(T^{n}\right)^{1 / n}=\min \left\{|\lambda|: \lambda \in \sigma_{\mathrm{ap}}(T)\right\} .
$$

An operator $T \in \mathcal{L}(\mathcal{H})$ is said to have the single-valued extension property if for every open set $U \subset \mathbb{C}$, the equation $(T-\lambda) f(\lambda)=0$ admits the zero function $f \equiv 0$ as a unique analytic solution on $U$. The local resolvent set, $\varrho_{T}(x)$, of $T$ at a point $x \in \mathcal{H}$ is the union of all open subsets $U \subset \mathbb{C}$ for which there exists an analytic function $f: U \rightarrow \mathcal{H}$ such that $(T-\lambda) f(\lambda)=x$ on $U$. The complement in $\mathbb{C}$ of $\varrho_{T}(x)$ is called the local spectrum of $T$ at $x$ and will be denoted by $\sigma_{T}(x)$. Recall that the local spectral radius of $T$ at $x$ is given by $r_{T}(x):=\lim _{\sup _{n \rightarrow \infty}}\left\|T^{n} x\right\|^{1 / n}$ and equals $\max \left\{|\lambda|: \lambda \in \sigma_{T}(x)\right\}$ whenever $T$ has the single-valued extension property. Moreover, if $T$ enjoys this property then for every $x \in \mathcal{H}$, there exists a unique maximal analytic solution $\widetilde{x}(\cdot)$ on $\varrho_{T}(x)$ for which $(T-\lambda) \widetilde{x}(\lambda)=x$ for all $\lambda \in \varrho_{T}(x)$, and which satisfies $\widetilde{x}(\lambda)=$ $-\sum_{n \geq 0} T^{n} x / \lambda^{n+1}$ on $\left\{\lambda \in \mathbb{C}:|\lambda|>r_{T}(x)\right\}$. If in addition $T$ is invertible, then $\widetilde{x}(\lambda)=\sum_{n \geq 1} \lambda^{n-1} T^{-n} x$ on the disc $\left\{\lambda \in \mathbb{C}:|\lambda|<1 / r_{T^{-1}}(x)\right\}$.

For a closed subset $F$ of $\mathbb{C}$, let

$$
\mathcal{H}_{T}(F):=\left\{x \in \mathcal{H}: \sigma_{T}(x) \subset F\right\}
$$

be the corresponding analytic spectral subspace; it is a $T$-hyperinvariant subspace, but is generally not closed in $\mathcal{H}$. The operator $T$ is said to satisfy Dunford's condition $(C)$ if for every closed subset $F$ of $\mathbb{C}$, the linear subspace $\mathcal{H}_{T}(F)$ is closed. Let $U$ be an open subset of $\mathbb{C}$ and let $\mathcal{O}(U, \mathcal{H})$ be the space of analytic $\mathcal{H}$-valued functions on $U$. Equipped with the topology of uniform convergence on compact subsets of $U$, the space $\mathcal{O}(U, \mathcal{H})$ is a Fréchet space. Note that every operator $T \in \mathcal{L}(\mathcal{H})$ induces a continuous mapping $T_{U}$ on $\mathcal{O}(U, \mathcal{H})$ defined by

$$
T_{U} f(\lambda)=(T-\lambda) f(\lambda) \quad \text { for } f \in \mathcal{O}(U, \mathcal{H}) \text { and } \lambda \in U .
$$

An operator $T \in \mathcal{L}(\mathcal{H})$ is said to have Bishop's property $(\beta)$ provided that, for every open subset $U$ of $\mathbb{C}$, the mapping $T_{U}$ is injective and has a closed range; equivalently, for every open subset $U$ in $\mathbb{C}$ and every sequence $\left(f_{n}\right)_{n}$ in $\mathcal{O}(U, H)$, the convergence of $\left(T_{U} f_{n}\right)_{n}$ to 0 in $\mathcal{O}(U, \mathcal{H})$ should entail the convergence of $\left(f_{n}\right)_{n}$ to 0 in $\mathcal{O}(U, \mathcal{H})$. It is known that hyponormal operators, $M$-hyponormal operators and more generally subscalar operators have Bishop's property $(\beta)$. It is also known that Bishop's property $(\beta)$ implies Dunford's condition $(C)$, and that the single-valued extension property follows from Dunford's condition $(C)$. 
An operator $T \in \mathcal{L}(\mathcal{H})$ is said to be decomposable if, for every open cover of $\mathbb{C}$ by two open subsets $U_{1}$ and $U_{2}$, there exist $T$-invariant closed linear subspaces $\mathcal{H}_{1}$ and $\mathcal{H}_{2}$ such that $\mathcal{H}=\mathcal{H}_{1}+\mathcal{H}_{2}$ and $\sigma\left(T_{\mid \mathcal{H}_{i}}\right) \subset U_{i}$ for $i=1,2$. Immediate examples of decomposable operators are provided by the normal operators and more generally by the spectral operators in the sense of Dunford. It is known that an operator $T \in \mathcal{L}(\mathcal{H})$ is decomposable if and only if both $T$ and $T^{*}$ have Bishop's property $(\beta)$. For thorough presentations of the local spectral theory, we refer to the monographs [12] and [13].

Throughout this paper, let $S$ be a weighted shift operator on $\mathcal{H}$ with a positive bounded weight sequence $\left(\omega_{n}\right)_{n}$, that is,

$$
S e_{n}=\omega_{n} e_{n+1} \quad \forall n,
$$

where $\left(e_{n}\right)_{n}$ is an orthonormal basis of $\mathcal{H}$. If the index $n$ runs over the non-negative integers (resp. all integers), then $S$ is called a unilateral (resp. bilateral) weighted shift.

Before proceeding, we would like to recall some simple but useful remarks which will be repeatedly used in what follows.

$\left(R_{1}\right) \quad$ For every $\alpha \in \mathbb{C},|\alpha|=1$, we have $(\alpha S) U_{\alpha}=U_{\alpha} S$, where $U_{\alpha}$ is the unitary operator defined on $\mathcal{H}$ by $U_{\alpha} e_{n}=\alpha^{n} e_{n}$ for all $n$.

$\left(R_{2}\right) \quad$ Let $K$ be a non-empty compact subset of $\mathbb{C}$. If $K$ is connected and has circular symmetry about the origin, then there are two real numbers $a$ and $b$ with $0 \leq a \leq b$ such that $K=\{\lambda \in \mathbb{C}: a \leq|\lambda| \leq b\}$.

$\left(R_{3}\right) \quad$ Let $T$ and $R$ in $\mathcal{L}(\mathcal{H})$ be such that $R$ is invertible. Then for every $x \in \mathcal{H}$, we have $\sigma_{T}(x)=\sigma_{R T R^{-1}}(R x)$.

Finally, we need to define the local functional calculus of operators having the single-valued extension property. This calculus was introduced by C. Apostol in [4] and has been studied by many authors (see [8], [9], [15], [18], and [23]). Let $U$ be an open subset of $\mathbb{C}$ and $K$ be a compact subset of $U$. Let $\left(\gamma_{i}\right)_{1 \leq i \leq n}$ be a finite family of disjoint closed rectifiable Jordan curves in $U \backslash K$. The formal sum $\gamma:=\gamma_{1}+\ldots+\gamma_{n}$ is called an oriented envelope of $K$ in $U$ if its winding number equals 1 on $K$ and 0 on $\mathbb{C} \backslash U$, i.e.,

$$
\eta_{\gamma}(\lambda):=\frac{1}{2 \pi i} \oint_{\gamma} \frac{1}{\alpha-\lambda} d \alpha= \begin{cases}1 & \text { for every } \lambda \in K, \\ 0 & \text { for every } \lambda \in \mathbb{C} \backslash U .\end{cases}
$$

Let $T \in \mathcal{L}(\mathcal{H})$, and let $v \in \mathcal{H}$. Assume that $T$ has the single-valued extension property. For an analytic complex-valued function $f$ on a neighborhood $U$ of $\sigma_{T}(v)$, we define a vector $f(T, v)$ by

$$
f(T, v):=\frac{-1}{2 \pi i} \oint_{\gamma} f(\lambda) \widetilde{v}(\lambda) d \lambda,
$$


where $\gamma$ is an oriented envelope of $\sigma_{T}(v)$ in $U$. Note that $f(T, v)$ is independent of the choice of $\gamma$. We end this introduction by quoting, without proof, the following theorem from [23] which will be used in what follows.

Theorem 1.1. Let $T \in \mathcal{L}(\mathcal{H})$, and let $v \in \mathcal{H}$. Assume that $T$ has the single-valued extension property. If $f$ is an analytic function on a neighborhood $O$ of $\sigma_{T}(v)$ and is not identically zero on each connected component of $O$, then:

(a) $Z_{T}(f, v):=\left\{\lambda \in \sigma_{T}(v): f(\lambda)=0\right\}$ is a finite set.

(b) $\sigma_{T}(v)=\sigma_{T}(f(T, v)) \cup Z_{T}(f, v)$.

Moreover, if $\sigma_{\mathrm{p}}(T)=\emptyset$, then $\sigma_{T}(v)=\sigma_{T}(f(T, v))$.

2. Preliminaries. In this section, we assemble some simple but useful results which will be needed. These results remain valid in the general setting of Banach space operators.

Let $\lambda_{0} \in \mathbb{C}$; recall that an operator $T \in \mathcal{L}(\mathcal{H})$ is said to have the singlevalued extension property (resp. Bishop's property $(\beta)$ ) at $\lambda_{0}$ if there is an open neighborhood $V$ of $\lambda_{0}$ such that for every open subset $U$ of $V$, the mapping $T_{U}$ is injective (resp. is injective and has a closed range). Obviously, $T$ has the single-valued extension property at $\lambda_{0}$ whenever $T$ has Bishop's property $(\beta)$ at $\lambda_{0}$. Note that $T$ has Bishop's property $(\beta)$ at $\lambda_{0}$ if and only if there is an open neighborhood $V$ of $\lambda_{0}$ such that for every open subset $U$ of $V$ and for every sequence $\left(f_{n}\right)_{n}$ in $\mathcal{O}(U, H)$, the convergence of $\left(T_{U} f_{n}\right)_{n}$ to 0 in $\mathcal{O}(U, \mathcal{H})$ entails the convergence of $\left(f_{n}\right)_{n}$ to 0 in $\mathcal{O}(U, \mathcal{H})$. Moreover, if $T$ has Bishop's property $(\beta)$ at any point $\lambda \in \mathbb{C}$ then $T$ has Bishop's classical property $(\beta)$.

For an arbitrary $T \in \mathcal{L}(\mathcal{H})$, we set

$$
\sigma_{\beta}(T):=\{\lambda \in \mathbb{C}: T \text { fails Bishop's property }(\beta) \text { at } \lambda\} .
$$

Note that since for every compact subset $K$ of $\mathbb{C} \backslash \sigma_{\text {ap }}(T)$ there is a positive constant $c=c(K)$ such that

$$
\|x\| \leq c\|(T-\lambda) x\|
$$

for all $x \in \mathcal{H}$ and $\lambda \in K$, we have the following known result.

Proposition 2.1. For any operator $T \in \mathcal{L}(\mathcal{H})$, the set $\sigma_{\beta}(T)$ is a closed subset of $\sigma_{\mathrm{ap}}(T)$.

An operator $T \in \mathcal{L}(\mathcal{H})$ is said to be cyclic if there exists a vector $x \in \mathcal{H}$, called a cyclic vector for $T$, such that

$$
\bigvee\left\{T^{n} x: n=0,1,2, \ldots\right\}=\mathcal{H}
$$

where $\bigvee$ denotes the closed linear span. We also say that $T$ is bi-cyclic with bi-cyclic vector $x \in \mathcal{H}$ if there exists a sequence $\left(x_{n}\right)_{n \geq 0}$ of elements of $\mathcal{H}$ 
such that $x_{0}=x, T x_{n+1}=x_{n}$ for all $n \geq 0$, and

$$
\bigvee\left\{x_{n}, T^{k} x: n, k=0,1,2, \ldots\right\}=\mathcal{H} .
$$

We note that each $x_{k}$ above is a bi-cyclic vector for $T$, and belongs to the hyper-range, $T^{\infty} \mathcal{H}:=\bigcap_{n \geq 0} T^{n} \mathcal{H}$, of $T$. In particular, the image, $\operatorname{ran} T$, of $T$ is dense in $\mathcal{H}$; or equivalently, $0 \notin \sigma_{\mathrm{p}}\left(T^{*}\right)$. Moreover, if $T$ is invertible, then it is easily seen that $x$ is a bi-cyclic vector for $T$ if and only if $x$ belongs to no non-trivial common closed invariant subspace of $T$ and its inverse $T^{-1}$. Immediate examples of cyclic and bi-cyclic operators are provided by scalar unilateral and bilateral weighted shift operators. If $S$ is a unilateral weighted shift, then $S$ is cyclic but not bi-cyclic and $e_{0}$ is a cyclic vector for $S$. If $S$ is a bilateral weighted shift, then each $e_{k}(k \in \mathbb{Z})$ is a bi-cyclic vector for $S$ but $S$ may or may not have cyclic vectors (see Proposition 42 of [20]).

For every operator $T \in \mathcal{L}(\mathcal{H})$, we set

$\Re(T):=\{\lambda \in \mathbb{C}: T$ fails the single-valued extension property at $\lambda\}$.

It is an open subset of $\mathbb{C}$ contained in $\sigma(T)$. The description of $\Re(T)$ for several operators $T$ can be found in [2] and [10]. Note that

$$
\overline{\sigma(T) \backslash \sigma_{\mathrm{ap}}(T)} \subset \Re\left(T^{*}\right)
$$

for every operator $T \in \mathcal{L}(\mathcal{H})$. This inclusion fails to be an equality in general: a counter-example can be found among scalar weighted shift operators. In [10], it is shown that if $T$ is a cyclic operator with Bishop's property $(\beta)$ then

$$
\Re\left(T^{*}\right)=\overline{\sigma(T) \backslash \sigma_{\mathrm{ap}}(T)} .
$$

This result also remains valid for every bi-cyclic operator with Bishop's property $(\beta)$. The proof is an adaptation of the one of Theorem 3.1 of [10] and will be omitted.

Proposition 2.2. Assume that $T \in \mathcal{L}(\mathcal{H})$ is a bi-cyclic operator. If $T$ has Bishop's property $(\beta)$, then $\Re\left(T^{*}\right)=\overline{\sigma(T) \backslash \sigma_{\text {ap }}(T)}$.

The following three lemmas are easy to verify. In fact, the next one is a particular case of Lemma 2 of [3] and the other two are trivial.

Lemma 2.3. Let $\mathcal{H}_{1}$ and $\mathcal{H}_{2}$ be complex Hilbert spaces, and let

$$
M_{C}=\left[\begin{array}{cc}
A & C \\
0 & B
\end{array}\right]
$$

be a $2 \times 2$ upper triangular operator matrix on $\mathcal{H}_{1} \oplus \mathcal{H}_{2}$. If both $A$ and $B$ have Bishop's property $(\beta)$ at a point $\lambda_{0} \in \mathbb{C}$, then so does $M_{C}$.

Proof. It is clear that, up to unitary equivalence, one has

$$
A \simeq M_{C \mid \mathcal{H}_{1}}, \quad B \simeq M_{C} / \mathcal{H}_{1} .
$$

Lemma 2 of [3] yields the desired result. 
Lemma 2.4. Let $\mathcal{H}_{1}$ and $\mathcal{H}_{2}$ be complex Hilbert spaces, and let

$$
M_{C}=\left[\begin{array}{cc}
A & C \\
0 & B
\end{array}\right]
$$

be a $2 \times 2$ upper triangular operator matrix on $\mathcal{H}_{1} \oplus \mathcal{H}_{2}$. If $B$ has the single-valued extension property, then $\sigma_{M_{C}}(x)=\sigma_{A}(x)$ for all $x \in \mathcal{H}_{1}$.

Lemma 2.5. If $S$ is a unilateral or bilateral weighted shift on $\mathcal{H}$, then $\sigma_{\beta}(S)$ has circular symmetry about the origin.

Proof. It is easy to see that if two operators $T \in \mathcal{L}(\mathcal{H})$ and $R \in \mathcal{L}(\mathcal{H})$ are similar, then $\sigma_{\beta}(T)=\sigma_{\beta}(R)$. In view of $\left(R_{1}\right)$, we have

$$
\sigma_{\beta}(S)=\sigma_{\beta}(\alpha S)=\alpha \sigma_{\beta}(S)
$$

for all $\alpha \in \mathbb{C}$ with $|\alpha|=1$, as desired.

3. Local spectral properties of unilateral weighted shift operators. In dealing with unilateral weighted shift operators, let $\left(\beta_{n}\right)_{n \geq 0}$ be the sequence given by

$$
\beta_{n}= \begin{cases}\omega_{0} \ldots \omega_{n-1} & \text { if } n>0 \\ 1 & \text { if } n=0,\end{cases}
$$

and let us associate to $S$ the following quantities:

$$
r_{2}(S):=\liminf _{n \rightarrow \infty} \beta_{n}^{1 / n}, \quad r_{3}(S):=\limsup _{n \rightarrow \infty} \beta_{n}^{1 / n} .
$$

Note that

$$
r(S)=\lim _{n \rightarrow \infty}\left[\sup _{k \geq 0} \frac{\beta_{n+k}}{\beta_{k}}\right]^{1 / n}, \quad r_{1}(S)=\lim _{n \rightarrow \infty}\left[\inf _{k \geq 0} \frac{\beta_{n+k}}{\beta_{k}}\right]^{1 / n},
$$

and

$$
m(S) \leq r_{1}(S) \leq r_{2}(S) \leq r_{3}(S) \leq r(S) \leq w(S) \leq\|S\| .
$$

Before going further, we note that since $\sigma_{\mathrm{p}}(S)=\emptyset$, the unilateral weighted shift $S$ always has the single-valued extension property.

3.1. Local spectra of unilateral weighted shift operators. The following result can be deduced from Proposition 1.6.9 of [13]; here we give a direct proof.

Proposition 3.1. For every non-zero $x \in \mathcal{H}$, we have

$$
\left\{\lambda \in \mathbb{C}:|\lambda| \leq r_{2}(S)\right\} \subset \sigma_{S}(x) .
$$

Proof. As $\bigcap_{n \geq 0} S^{n} \mathcal{H}=\{0\}$, we see that $0 \in \sigma_{S}(x)$ for every $x \in \mathcal{H} \backslash\{0\}$. Thus, we may assume that $r_{2}(S)>0$. Let $O:=\left\{\lambda \in \mathbb{C}:|\lambda|<r_{2}(S)\right\}$, and consider the following analytic $\mathcal{H}$-valued function on $O$ :

$$
k(\lambda):=\sum_{n=0}^{\infty} \frac{\lambda^{n}}{\beta_{n}} e_{n}
$$


It is easy to see that $(S-\lambda)^{*} k(\bar{\lambda})=0$ for every $\lambda \in O$. Now, let $x=$ $\sum_{n \geq 0} a_{n} e_{n} \in \mathcal{H}$ be such that $O \cap \varrho_{S}(x) \neq \emptyset$. So, for every $\lambda \in O \cap \varrho_{S}(x)$, we have

$$
\widehat{x}(\lambda):=\langle x ; k(\bar{\lambda})\rangle=\langle(S-\lambda) \widetilde{x}(\lambda) ; k(\bar{\lambda})\rangle=\left\langle\widetilde{x}(\lambda) ;(S-\lambda)^{*} k(\bar{\lambda})\right\rangle=0 .
$$

Since $\widehat{x}(\lambda)=\sum_{n=0}^{\infty}\left(a_{n} / \beta_{n}\right) \lambda^{n} e_{n}$ for every $\lambda \in O$, we see that $a_{n}=0$ for every $n \geq 0$, and therefore, $x=0$. Thus, the proof is complete.

Corollary 3.2. For every $x \in \mathcal{H}, \sigma_{S}(x)$ is a connected set.

Proof. Suppose that there is a non-zero $x \in \mathcal{H}$ such that $\sigma_{S}(x)$ is disconnected. So, there are two non-empty disjoint compact subsets $\sigma_{1}$ and $\sigma_{2}$ of $\mathbb{C}$ such that $\sigma_{S}(x)=\sigma_{1} \cup \sigma_{2}$. It follows from Proposition 1.2.16(g) of [13] that $\mathcal{H}_{S}\left(\sigma_{S}(x)\right)=\mathcal{H}_{S}\left(\sigma_{1}\right) \oplus \mathcal{H}_{S}\left(\sigma_{2}\right)$. Therefore, there are two non-zero elements $x_{1}$ and $x_{2}$ of $\mathcal{H}$ such that $x=x_{1}+x_{2}$ and $\sigma_{S}\left(x_{i}\right) \subset \sigma_{i}, i=1,2$. In particular, $\sigma_{S}\left(x_{1}\right) \cap \sigma_{S}\left(x_{2}\right)=\emptyset$, which contradicts Proposition 3.1.

We now give a simple proof of Theorem 4 of [20] from the point of view of the local spectral theory.

Corollary 3.3. The spectrum of $S$ is the disc $\{\lambda \in \mathbb{C}:|\lambda| \leq r(S)\}$.

Proof. By Proposition 1.3.2 of [13], we have $\sigma(S)=\bigcup_{x \in \mathcal{H} \backslash\{0\}} \sigma_{S}(x)$. As each $\sigma_{S}(x)$ is connected, and $\left\{\lambda \in \mathbb{C}:|\lambda| \leq r_{2}(S)\right\} \subset \bigcap_{x \in \mathcal{H} \backslash\{0\}} \sigma_{S}(x) \neq \emptyset$ (see Proposition 3.1), we deduce that $\sigma(S)$ is also a connected set containing the disc $\left\{\lambda \in \mathbb{C}:|\lambda| \leq r_{2}(S)\right\}$. On the other hand, from $\left(R_{1}\right)$ we see that $\sigma(S)$ has circular symmetry about the origin. So, by $\left(R_{2}\right), \sigma(S)$ must be the $\operatorname{disc}\{\lambda \in \mathbb{C}:|\lambda| \leq r(S)\}$.

REMARK 3.4. Let $x=\sum_{n \geq 0} a_{n} e_{n}$ be a non-zero element of $\mathcal{H}$. It follows from Proposition 3.1 that $r_{2}(S) \leq r_{S}(x)$. In fact, more can be shown:

$$
r_{3}(S) \leq r_{S}(x) \leq r(S) .
$$

Indeed, for every integer $n \geq 0$, we have

$$
S^{n} x=\sum_{k \geq 0} a_{k} S^{n} e_{k}=\sum_{k \geq 0} a_{k} \frac{\beta_{n+k}}{\beta_{k}} e_{n+k} .
$$

Since there is an integer $k_{0} \geq 0$ such that $a_{k_{0}} \neq 0$, it then follows that

$$
\left|a_{k_{0}}\right| \frac{\beta_{n+k_{0}}}{\beta_{k_{0}}} \leq\left[\sum_{k \geq 0}\left|a_{k}\right|^{2}\left|\frac{\beta_{n+k}}{\beta_{k}}\right|^{2}\right]^{1 / 2}=\left\|S^{n} x\right\|, \quad \forall n \geq 0 .
$$

Now, by taking the $n$th root and then $\lim \sup$ as $n \rightarrow \infty$, we get $r_{3}(S) \leq$ $r_{S}(x)$.

Let $x$ be a non-zero element of $\mathcal{H}$; from Corollary 3.2, we see that every circle of radius $r, 0 \leq r \leq r_{S}(x)$, intersects $\sigma_{S}(x)$. So, in view of Remark 3.4, $\sigma_{S}(x)$ may contain points which are not in the $\operatorname{disc}\left\{\lambda \in \mathbb{C}:|\lambda| \leq r_{2}(S)\right\}$. 
The next result gives a complete description of the local spectrum of $S$ at most of the points in $\mathcal{H}$, and refines the local spectral inclusion of Proposition 3.1. For every non-zero $x=\sum_{n \geq 0} a_{n} e_{n} \in \mathcal{H}$, we set

$$
R_{\omega}(x):=\liminf _{n \rightarrow \infty}\left|\frac{\beta_{n}}{a_{n}}\right|^{1 / n} .
$$

Note that $r_{2}(S) \leq R_{\omega}(x) \leq \infty$ for every non-zero $x \in \mathcal{H}$.

TheOREM 3.5. For every non-zero $x=\sum_{n \geq 0} a_{n} e_{n} \in \mathcal{H}$, we have:

(a) If $r_{3}(S)<R_{\omega}(x)$, then $\sigma_{S}(x)=\left\{\lambda \in \mathbb{C}:|\lambda| \leq r_{3}(S)\right\}$.

(b) If $r_{3}(S) \geq R_{\omega}(x)$, then $\left\{\lambda \in \mathbb{C}:|\lambda| \leq R_{\omega}(x)\right\} \subset \sigma_{S}(x)$.

Proof. Let us first show that

$$
\sigma_{S}\left(e_{0}\right)=\left\{\lambda \in \mathbb{C}:|\lambda| \leq r_{3}(S)\right\} .
$$

Let $\alpha \in \mathbb{C},|\alpha|=1$; by $\left(R_{1}\right)$, we have $\alpha S=U_{\alpha} S U_{\alpha}^{*}$, and so $\sigma_{S}\left(e_{0}\right)=$ $\sigma_{\alpha S}\left(U_{\alpha} e_{0}\right)$, by $\left(R_{3}\right)$; hence, $\sigma_{S}\left(e_{0}\right)=\alpha \sigma_{S}\left(e_{0}\right)$. This shows that $\sigma_{S}\left(e_{0}\right)$ has circular symmetry about the origin. As $\sigma_{S}\left(e_{0}\right)$ is a connected set containing the disc $\left\{\lambda \in \mathbb{C}:|\lambda| \leq r_{2}(S)\right\}$, it follows from $\left(R_{2}\right)$ that $\sigma_{S}\left(e_{0}\right)$ must be the $\operatorname{disc}\left\{\lambda \in \mathbb{C}:|\lambda| \leq r_{S}\left(e_{0}\right)\right\}$. Therefore, (3.2) holds, since $r_{S}\left(e_{0}\right)=r_{3}(S)$.

Next, keep in mind that for every $\lambda \in \varrho_{S}\left(e_{0}\right)=\left\{\lambda \in \mathbb{C}:|\lambda|>r_{3}(S)\right\}$, we have

$$
\widetilde{e}_{0}(\lambda)=-\sum_{n \geq 0} \frac{S^{n} e_{0}}{\lambda^{n+1}}=-\sum_{n \geq 0} \frac{\beta_{n}}{\lambda^{n+1}} e_{n} .
$$

Now, we are able to prove the first statement.

(a) Suppose that $r_{3}(S)<R_{\omega}(x)$. Note that the function $f(\lambda):=$ $\sum_{n \geq 0}\left(a_{n} / \beta_{n}\right) \lambda^{n}$ is analytic on the neighborhood $\left\{\lambda \in \mathbb{C}:|\lambda|<R_{\omega}(x)\right\}$ of $\sigma_{S}\left(e_{0}\right)$. Let $r$ be a real number such that $r_{3}(S)<r<R_{\omega}(x)$. We have

$$
\begin{aligned}
f\left(S, e_{0}\right) & =\frac{-1}{2 \pi i} \oint_{|\lambda|=r} f(\lambda) \widetilde{e}_{0}(\lambda) d \lambda=\frac{-1}{2 \pi i} \oint_{|\lambda|=r} f(\lambda)\left[-\sum_{n \geq 0} \frac{\beta_{n}}{\lambda^{n+1}} e_{n}\right] d \lambda \\
& =\sum_{n \geq 0}\left[\frac{1}{2 \pi i} \oint_{|\lambda|=r} \frac{f(\lambda)}{\lambda^{n+1}} d \lambda\right] \beta_{n} e_{n}=x
\end{aligned}
$$

and so, by Theorem 1.1,

$$
\sigma_{S}(x)=\sigma_{S}\left(e_{0}\right)=\left\{\lambda \in \mathbb{C}:|\lambda| \leq r_{3}(S)\right\} .
$$

(b) Suppose that $r_{3}(S) \geq R_{\omega}(x)$. If $r_{2}(S)=R_{\omega}(x)$, then according to Proposition 3.1 there is nothing to prove; thus we may suppose, in particular, 
that $0<R_{\omega}(x)$. For each $n \geq 0$, let

$$
\begin{aligned}
& A_{n}(\lambda):=-\frac{a_{0}}{\lambda^{n+1}} \beta_{n}-\frac{a_{1}}{\lambda^{n}} \frac{\beta_{n}}{\beta_{1}}-\frac{a_{2}}{\lambda^{n-1}} \frac{\beta_{n}}{\beta_{2}}-\ldots-\frac{a_{n}}{\lambda}, \quad \lambda \in \mathbb{C} \backslash\{0\}, \\
& B_{n}(\lambda):=a_{0}+\frac{a_{1}}{\beta_{1}} \lambda+\frac{a_{2}}{\beta_{2}} \lambda^{2}+\ldots+\frac{a_{n}}{\beta_{n}} \lambda^{n}, \quad \lambda \in \mathbb{C} .
\end{aligned}
$$

We have

$$
A_{n}(\lambda)=-\frac{\beta_{n}}{\lambda^{n+1}} B_{n}(\lambda), \quad \lambda \in \mathbb{C} \backslash\{0\} .
$$

By writing $\widetilde{x}(\lambda):=\sum_{n \geq 0} \widetilde{A}_{n}(\lambda) e_{n}, \lambda \in \varrho_{S}(x)$, we deduce from the equation

$$
(S-\lambda) \widetilde{x}(\lambda)=x, \quad \lambda \in \varrho_{S}(x),
$$

that for every $\lambda \in \varrho_{S}(x)$, we have

$$
\left\{\begin{array}{l}
-\lambda \widetilde{A}_{0}(\lambda)=a_{0}, \\
\widetilde{A}_{n}(\lambda) \omega_{n}-\lambda \widetilde{A}_{n+1}(\lambda)=a_{n+1} \quad \text { for every } n \geq 0 .
\end{array}\right.
$$

Therefore, for every $n \geq 0$,

$$
\widetilde{A}_{n}(\lambda)=A_{n}(\lambda)=-\frac{a_{0}}{\lambda^{n+1}} \beta_{n}-\frac{a_{1}}{\lambda^{n}} \frac{\beta_{n}}{\beta_{1}}-\frac{a_{2}}{\lambda^{n-1}} \frac{\beta_{n}}{\beta_{2}}-\ldots-\frac{a_{n}}{\lambda}, \quad \lambda \in \varrho_{S}(x) .
$$

Since $\|\widetilde{x}(\lambda)\|^{2}=\sum_{n \geq 0}\left|\widetilde{A}_{n}(\lambda)\right|^{2}=\sum_{n \geq 0}\left|A_{n}(\lambda)\right|^{2}<\infty$ for every $\lambda \in \varrho_{S}(x)$, it follows that

$$
\lim _{n \rightarrow \infty} A_{n}(\lambda)=0 \quad \text { for every } \lambda \in \varrho_{S}(x) .
$$

We shall show that (3.4) is not satisfied for most of the points in the nonempty open disc $V(x):=\left\{\lambda \in \mathbb{C}:|\lambda|<R_{\omega}(x)\right\}$. It is clear that the sequence $\left(B_{n}\right)_{n \geq 0}$ converges uniformly on compact subsets of $V(x)$ to the non-zero power series $B(\lambda)=\sum_{n \geq 0}\left(a_{n} / \beta_{n}\right) \lambda^{n}$. Now, let $\lambda_{0} \in V(x) \backslash\{0\}$ be such that $B\left(\lambda_{0}\right) \neq 0$; there is $\varepsilon>0$ and an integer $n_{0}$ such that $\varepsilon<\left|B_{n}\left(\lambda_{0}\right)\right|$ for every $n \geq n_{0}$. On the other hand, $\left|\lambda_{0}\right|<r_{3}(S)$, so there is a subsequence $\left(n_{k}\right)_{k \geq 0}$ of integers greater than $n_{0}$ such that $\left|\lambda_{0}\right|^{n_{k}}<\beta_{n_{k}}$. Thus, (3.3) gives

$$
\left|A_{n_{k}}\left(\lambda_{0}\right)\right|=\left|-\frac{\beta_{n_{k}}}{\lambda_{0}^{n_{k}+1}} B_{n_{k}}\left(\lambda_{0}\right)\right| \geq \frac{\varepsilon}{\left|\lambda_{0}\right|} \quad \text { for every } k \geq 0 .
$$

Hence, by (3.4), $\lambda_{0} \notin \varrho_{S}(x)$. Since the set of zeros of $B$ is at most countable, we have $\left\{\lambda \in \mathbb{C}:|\lambda| \leq R_{\omega}(x)\right\} \subset \sigma_{S}(x)$.

REMARK 3.6. Let $\mathcal{H}_{0}$ denote all the finite linear combinations of the vectors $e_{n}(n \geq 0)$; it is clearly a dense subspace of $\mathcal{H}$. It follows from Theorem 3.5(a) that for every non-zero $x \in \mathcal{H}_{0}$, we have

$$
\sigma_{S}(x)=\left\{\lambda \in \mathbb{C}:|\lambda| \leq r_{3}(S)\right\}
$$

This can also be deduced from Theorem 1.1 and (3.2), since for every nonzero $x \in \mathcal{H}_{0}$, there is a non-zero polynomial $p$ such that $x=p(S) e_{0}$. 
COROLlary 3.7. If $T \in \mathcal{L}(\mathcal{H})$ is a non-zero operator commuting with $S$ then

$$
\sigma_{S}\left(T e_{n}\right)=\left\{\lambda \in \mathbb{C}:|\lambda| \leq r_{3}(S)\right\} \quad \text { for all } n \geq 0 .
$$

Proof. We shall first show that

$$
\sigma_{S}\left(T e_{0}\right)=\left\{\lambda \in \mathbb{C}:|\lambda| \leq r_{3}(S)\right\} .
$$

By writing $T e_{0}:=\sum_{n \geq 0} a_{n} e_{n}$, we see that for all $n, k \geq 0$ we have

$$
\frac{a_{n}}{\beta_{n}} \beta_{n+k}=\left\langle T e_{0} ; S^{* k} e_{n+k}\right\rangle=\left\langle T S^{k} e_{0} ; e_{n+k}\right\rangle=\beta_{k}\left\langle T e_{k} ; e_{n+k}\right\rangle .
$$

This gives

$$
\frac{\beta_{n+k}}{\beta_{k}} \leq\|T\|\left|\frac{\beta_{n}}{a_{n}}\right| \quad \text { for all } n, k \geq 0 .
$$

Thus $r(S) \leq R_{\omega}\left(T e_{0}\right)$, and the desired identity follows from Theorem 3.5.

Now, let $n$ be an arbitrary integer. As $\left(1 / \beta_{n}\right) T S^{n}$ is a non-zero operator commuting with $S$ and $e_{n}=\left(1 / \beta_{n}\right) S^{n} e_{0}$, it follows from what has been shown above that

$$
\sigma_{S}\left(T e_{n}\right)=\sigma_{S}\left(\left(1 / \beta_{n}\right) T S^{n} e_{0}\right)=\left\{\lambda \in \mathbb{C}:|\lambda| \leq r_{3}(S)\right\} .
$$

This finishes the proof.

Question 1. Does $\sigma_{S}(x)$ equal $\left\{\lambda \in \mathbb{C}:|\lambda| \leq r_{S}(x)\right\}$ for every non-zero $x \in \mathcal{H}$ ?

In view of Proposition 3.1 and (3.1), an interesting special case of this question suggests itself.

Question 2. Is $\left\{\lambda \in \mathbb{C}:|\lambda| \leq r_{3}(S)\right\}$ contained in $\sigma_{S}(x)$ for every non-zero $x \in \mathcal{H}$ ?

3.2. Which unilateral weighted shift operators satisfy Dunford's condition $(C)$ ? The following result gives a necessary condition for a unilateral weighted shift operator $S$ to enjoy Dunford's condition $(C)$.

THEOREM 3.8. If $S$ satisfies Dunford's condition $(C)$, then $r(S)=r_{3}(S)$, so that

$$
\sigma_{S}\left(T e_{n}\right)=\sigma(S)=\{\lambda \in \mathbb{C}:|\lambda| \leq r(S)\} \quad(n \geq 0)
$$

for every non-zero operator $T \in \mathcal{L}(\mathcal{H})$ that commutes with $S$.

Proof. Suppose that $S$ satisfies Dunford's condition $(C)$, and let $F:=$ $\left\{\lambda \in \mathbb{C}:|\lambda| \leq r_{3}(S)\right\}$. It follows from Theorem 3.5(a) that $\mathcal{H}_{S}(F)$ contains a dense subset of $\mathcal{H}$. As $\mathcal{H}_{S}(F)$ is closed, we get $\mathcal{H}_{S}(F)=\mathcal{H}$; this means that $\sigma_{S}(x) \subset F$ for every $x \in \mathcal{H}$. It follows that $\sigma(S)=\bigcup_{x \in \mathcal{H}} \sigma_{S}(x) \subset F$ (see Proposition 1.3.2 of [13]) and hence that $r_{3}(S) \leq r(S) \leq r_{3}(S)$ as desired. The remaining assertion is now an immediate consequence of Corollary 3.7. 
An interesting special case occurs when the sequence $\left(\beta_{n}^{1 / n}\right)_{n \geq 1}$ converges. Then by combining Proposition 3.1 and Theorem 3.8, we see that the following statements are equivalent.

(a) $\sigma_{S}(x)=\sigma(S)$ for every non-zero $x \in \mathcal{H}$.

(b) $S$ satisfies Dunford's condition $(C)$.

(c) $r(S)=\lim _{n \rightarrow \infty} \beta_{n}^{1 / n}$.

In the general case, we note that if $r(S)=r_{3}(S)$, then $\sigma_{S}(x)=\sigma(S)$ for most of the points $x$ in $\mathcal{H}$. Thus, we conjecture that the following statements are equivalent.

(a) $\sigma_{S}(x)=\sigma(S)$ for every non-zero $x \in \mathcal{H}$.

(b) $S$ satisfies Dunford's condition $(C)$.

(c) $r(S)=r_{3}(S)$.

Note that a positive answer to Question 2 will prove this conjecture.

3.3. Which unilateral weighted shift operators have Bishop's property $(\beta)$ ? In [16], T. L. Miller and V. G. Miller have shown that if $r_{1}(S)<r_{2}(S)$, then $S$ fails Bishop's property $(\beta)$. In fact, this result could be obtained by combining Theorem 3.1 of [24] and Theorem 10(ii) of [20]. But the proof given in [16] is simple and elegant. Here, we refine this result as follows.

THEOREM 3.9. If $S$ has Bishop's property $(\beta)$, then $r_{1}(S)=r(S)$. Conversely, if $r_{1}(S)=r(S)$, then either $S$ has Bishop's property $(\beta)$, or $\sigma_{\beta}(S)=$ $\{\lambda \in \mathbb{C}:|\lambda|=r(S)\}$.

Proof. Suppose that $S$ has Bishop's property $(\beta)$. According to the above discussion, we have

$$
r_{1}(S)=r_{2}(S)
$$

On the other hand, it follows from [5] that $S$ is power-regular, that is, the sequence $\left(\left\|S^{n} x\right\|^{1 / n}\right)_{n \geq 0}$ converges for all $x \in \mathcal{H}$. In particular, $\left(\left\|S^{n} e_{0}\right\|^{1 / n}\right)_{n \geq 0}$ converges; so,

$$
r_{2}(S)=r_{3}(S)
$$

As $S$ satisfies Dunford's condition $(C)$, we see from Theorem 3.8 that

$$
r_{3}(S)=r(S) \text {. }
$$

Therefore, the result follows from (3.5)-(3.7).

Conversely, suppose that $r_{1}(S)=r(S)$. Since $\sigma_{\text {ap }}(S)=\{\lambda \in \mathbb{C}:|\lambda|=$ $r(S)\}$ (see Theorem 1 of [19] or Theorem 6 of [20]), the desired result holds by combining Proposition 2.1 and Lemma 2.5.

In view of Theorem 3.9, the following question suggests itself.

Question 3. Suppose that $r_{1}(S)=r(S)$. Does $S$ have Bishop's prop$\operatorname{erty}(\beta)$ ? 
The next two propositions give a condition on the weights $\left(\omega_{n}\right)_{n \geq 0}$ for which the corresponding weighted shift has Bishop's property $(\beta)$.

Proposition 3.10. If $m(S)=w(S)$, then $S$ has Bishop's property $(\beta)$.

Proof. It is well known that for every $T \in \mathcal{L}(\mathcal{H})$, and every $\lambda$ not in the closure of $W(T)$, the norm of the resolvent $(T-\lambda)^{-1}$ admits the estimate

$$
\left\|(T-\lambda)^{-1}\right\| \leq \frac{1}{\operatorname{dist}(\lambda, W(T))} .
$$

If $m(S)=w(S)=0$, then $S$ is quasi-nilpotent, and so, by Proposition 1.6.14 of [13], decomposable. Therefore, $S$ has Bishop's property $(\beta)$. Now, without loss of generality suppose that $m(S)=w(S)=1$. It follows from Proposition 16 of [20] that

$$
\{\lambda \in \mathbb{C}:|\lambda|<1\} \subset W(S) \subset\{\lambda \in \mathbb{C}:|\lambda| \leq 1\} .
$$

By (3.8) we have

$$
\left\|(S-\lambda)^{-1}\right\| \leq \frac{1}{|1-| \lambda||} \quad \text { for every } \lambda \in \mathbb{C},|\lambda|>1 .
$$

This shows that, for every $x \in \mathcal{H}$,

$$
|1-| \lambda||\|x\| \leq\|(S-\lambda) x\| \quad \text { for all } \lambda \in \mathbb{C},|\lambda|>1 .
$$

On the other hand, for every $x \in \mathcal{H}$ and $\lambda \in \mathbb{C},|\lambda|<1$, we have

$$
\|(S-\lambda) x\| \geq\|S x\|-|\lambda|\|x\| \geq m(S)\|x\|-|\lambda|\|x\| \geq(1-|\lambda|)\|x\| .
$$

Therefore, for every $\lambda \in \mathbb{C} \backslash\{\mu \in \mathbb{C}:|\mu|=1\}$ and every $x \in \mathcal{H}$, we have

$$
|1-| \lambda||\|x\| \leq\|(S-\lambda) x\| .
$$

Now, Theorem 1.7.1 of [13] implies that $S$ has Bishop's property $(\beta)$.

The next example gives a collection of unilateral weighted shift operators with Bishop's property $(\beta)$ which shows that the assumption $m(S)=w(S)$ in Proposition 3.10 is not necessary.

EXAMPLE 3.11. Let $a$ be a positive real number, and let $S_{a}$ be the unilateral weighted shift with weights $\left(\omega_{n}(a)\right)_{n \geq 0}$ given by

$$
\omega_{n}(a)= \begin{cases}a & \text { if } n=0 \\ 1 & \text { if } n>0\end{cases}
$$

It is clear that $m\left(S_{a}\right)=\min (1, a)$, and $S_{a}$ has Bishop's property $(\beta)$ since it is similar to the unweighted shift operator (see Theorem 2 of [20]). On the other hand, it was shown in [7] (see also Proposition 2 of [21]) that:

(a) If $a<1$, then $w\left(S_{a}\right)=1>m\left(S_{a}\right)=a$.

(b) If $1 \leq a \leq \sqrt{2}$, then $w\left(S_{a}\right)=m\left(S_{a}\right)=1$.

(c) If $\sqrt{2}<a$, then $w\left(S_{a}\right)=a^{2} /\left(2 \sqrt{a^{2}-1}\right)>m\left(S_{a}\right)=1$. 
Proposition 3.12. If $\left(\omega_{n}\right)_{n \geq 0}$ is a periodic sequence, then $S$ has Bishop's property $(\beta)$.

Proof. Suppose that $\left(\omega_{n}\right)_{n \geq 0}$ has period $k$. Then, for every $x \in \mathcal{H}$, we have $\left\|S^{k} x\right\|=\omega_{0} \ldots \omega_{k-1}\|x\|$. Hence, $\frac{1}{\omega_{0} \ldots \omega_{k-1}} S^{k}$ is an isometry; so, by Proposition 1.6.7 of [13], $S^{k}$ has Bishop's property $(\beta)$, and Theorem 3.3.9 of [13] implies that so does $S$.

Recall that the weight $\left(\omega_{n}\right)_{n \geq 0}$ is said to be almost periodic if there is a periodic positive sequence $\left(p_{n}\right)_{n \geq 0}$ such that $\lim _{n \rightarrow \infty}\left(\omega_{n}-p_{n}\right)=0$. Note that if $k$ is the period of $\left(p_{n}\right)_{n \geq 0}$, then

$$
r_{1}(S)=r(S)=\left(p_{0} \ldots p_{k-1}\right)^{1 / k} .
$$

This suggests a weaker version of Question 3.

QUestion 4. Suppose that the weight $\left(\omega_{n}\right)_{n \geq 0}$ is almost periodic. Does $S$ have Bishop's property $(\beta)$ ?

\section{Local spectral properties of bilateral weighted shift opera-} tors. In dealing with bilateral weighted shift operators, let $\left(\beta_{n}\right)_{n \in \mathbb{Z}}$ be the sequence given by

$$
\beta_{n}= \begin{cases}\omega_{0} \ldots \omega_{n-1} & \text { if } n>0 \\ \frac{1}{\omega_{n} \ldots \omega_{-1}} & \text { if } n<0\end{cases}
$$

and set

$$
\begin{aligned}
& r^{-}(S)=\lim _{n \rightarrow \infty}\left[\sup _{k>0} \frac{\beta_{-k}}{\beta_{-n-k}}\right]^{1 / n}, \quad r^{+}(S)=\lim _{n \rightarrow \infty}\left[\sup _{k \geq 0} \frac{\beta_{n+k}}{\beta_{k}}\right]^{1 / n}, \\
& r_{1}^{-}(S)=\lim _{n \rightarrow \infty}\left[\inf _{k>0} \frac{\beta_{-k}}{\beta_{-n-k}}\right]^{1 / n}, \quad r_{1}^{+}(S)=\lim _{n \rightarrow \infty}\left[\inf _{k \geq 0} \frac{\beta_{n+k}}{\beta_{k}}\right]^{1 / n}, \\
& r_{2}^{-}(S)=\liminf _{n \rightarrow \infty} \beta_{-n}^{-1 / n}, \\
& r_{3}^{-}(S)=\limsup _{n \rightarrow \infty} \beta_{-n}^{-1 / n}, \quad r_{3}^{+}(S)=\limsup _{n \rightarrow \infty} \beta_{n}^{1 / n} .
\end{aligned}
$$

Note that

$$
\begin{array}{llrl}
r_{1}(S) & =\min \left(r_{1}^{-}(S), r_{1}^{+}(S)\right), & & r(S)=\max \left(r^{-}(S), r^{+}(S)\right), \\
r_{1}^{-}(S) \leq r_{2}^{-}(S) \leq r_{3}^{-}(S) \leq r^{-}(S), & r_{1}^{+}(S) \leq r_{2}^{+}(S) \leq r_{3}^{+}(S) \leq r^{+}(S) .
\end{array}
$$

4.1. Local spectra of bilateral weighted shift operators. We begin by pointing out that there are bilateral weighted shift operators which do not have the single-valued extension property. The following result, which can be easily proved, gives a necessary and sufficient condition for a bilateral weighted shift operator $S$ to enjoy this property. 
Proposition 4.1. S has the single-valued extension property if and only if $r_{2}^{-}(S) \leq r_{3}^{+}(S)$.

Proof. If $r_{2}^{-}(S) \leq r_{3}^{+}(S)$, then according to Theorem 9 of $[20], \sigma_{\mathrm{p}}(S)$ has empty interior. Hence, $S$ has the single-valued extension property.

Conversely, suppose that $r_{3}^{+}(S)<r_{2}^{-}(S)$. Let

$$
O:=\left\{\lambda \in \mathbb{C}: r_{3}^{+}(S)<|\lambda|<r_{2}^{-}(S)\right\}
$$

It is easy to see that the analytic $\mathcal{H}$-valued function on $O$ defined by

$$
f(\lambda):=\sum_{n \in \mathbb{Z}} \frac{\beta_{n}}{\lambda^{n}} e_{n}
$$

satisfies the equation

$$
(S-\lambda) f(\lambda)=0, \quad \lambda \in O .
$$

This shows that $S$ does not have the single-valued extension property.

COROLlary 4.2. $S^{*}$ has the single-valued extension property if and only if $r_{2}^{+}(S) \leq r_{3}^{-}(S)$.

Proof. As $S^{*}$ is also a bilateral weighted shift operator with $r_{2}^{-}\left(S^{*}\right)=$ $r_{2}^{+}(S)$, and $r_{3}^{+}\left(S^{*}\right)=r_{3}^{-}(S)$, this is a direct consequence of Proposition 4.1.

An analogue of Proposition 3.1 is

Proposition 4.3. For every non-zero $x \in \mathcal{H}$, we have

$$
\left\{\lambda \in \mathbb{C}: r_{3}^{-}(S)<|\lambda|<r_{2}^{+}(S)\right\} \subset \sigma_{S}(x) .
$$

Proof. If $r_{2}^{+}(S) \leq r_{3}^{-}(S)$, then there is nothing to prove. Assume that $r_{3}^{-}(S)<r_{2}^{+}(S)$, and set

$$
O:=\left\{\lambda \in \mathbb{C}: r_{3}^{-}(S)<|\lambda|<r_{2}^{+}(S)\right\} .
$$

Now, consider the analytic $\mathcal{H}$-valued function $k$ on $O$ defined by

$$
k(\lambda):=\sum_{n \in \mathbb{Z}} \frac{\lambda^{n}}{\beta_{n}} e_{n} .
$$

We have $(S-\lambda)^{*} k(\bar{\lambda})=0$ for every $\lambda \in O$. The rest of the proof goes as in the proof of Proposition 3.1.

Corollary 4.4. If $r_{3}^{-}(S)<r_{2}^{+}(S)$, then each $\sigma_{S}(x)$ is connected.

Proof. The proof is similar to the proof of Corollary 3.2.

REMARK 4.5. A similar proof to that of (3.1) yields

$$
r_{3}^{+}(S) \leq r_{S}(x) \leq r(S) \quad \text { for every non-zero } x \in \mathcal{H} .
$$

If $S$ is invertible, then $S^{-1}$ is also a bilateral weighted shift with weights $\left(1 / \omega_{-n-1}\right)_{n \in \mathbb{Z}}$. So, by applying $(4.9)$ to $S^{-1}$, we get

$$
1 / r_{2}^{-}(S) \leq r_{S^{-1}}(x) \leq r\left(S^{-1}\right) \quad \text { for every non-zero } x \in \mathcal{H} .
$$


Let $\mathcal{H}_{0}$ denote all finite linear combinations of the vectors $e_{n}(n \in \mathbb{Z})$. The following proposition gives a complete description of the local spectrum of $S$ at a point $x \in \mathcal{H}_{0}$.

Proposition 4.6. For every non-zero $x \in \mathcal{H}_{0}$, we have

$$
\sigma_{S}(x)=\left\{\lambda \in \mathbb{C}: r_{2}^{-}(S) \leq|\lambda| \leq r_{3}^{+}(S)\right\} .
$$

Proof. First, let us prove that

$$
\sigma_{S}\left(e_{n}\right)=\left\{\lambda \in \mathbb{C}: r_{2}^{-}(S) \leq|\lambda| \leq r_{3}^{+}(S)\right\}, \quad \forall n \in \mathbb{Z} .
$$

We limit ourselves to the case $n=0$; the proof for arbitrary $n \in \mathbb{Z}$ is similar.

Let $O_{1}:=\left\{\lambda \in \mathbb{C}:|\lambda|>r_{3}^{+}(S)\right\}$, and consider the $\mathcal{H}$-valued function on $O_{1}$ defined by

$$
f_{1}(\lambda):=\sum_{n=0}^{\infty} \frac{\beta_{n}}{\lambda^{n+1}} e_{n} .
$$

As $(S-\lambda) f_{1}(\lambda)=e_{0}$ for every $\lambda \in O_{1}$, we note that

$$
\sigma_{S}\left(e_{0}\right) \subset\left\{\lambda \in \mathbb{C}:|\lambda| \leq r_{3}^{+}(S)\right\} .
$$

We also have

$$
\sigma_{S}\left(e_{0}\right) \subset\left\{\lambda \in \mathbb{C}:|\lambda| \geq r_{2}^{-}(S)\right\} .
$$

Indeed, we may assume $r_{2}^{-}(S)>0$. The $\mathcal{H}$-valued function on $O_{2}:=\{\lambda \in \mathbb{C}$ : $\left.|\lambda|<r_{2}^{-}(S)\right\}$ defined by

$$
f_{2}(\lambda):=\sum_{n=1}^{\infty} \lambda^{n-1} \beta_{-n} e_{-n}
$$

satisfies $(S-\lambda) f_{2}(\lambda)=e_{0}$ for every $\lambda \in O_{2}$. This proves (4.13). It follows from (4.12) and (4.13) that

$$
\sigma_{S}\left(e_{0}\right) \subset\left\{\lambda \in \mathbb{C}: r_{2}^{-}(S) \leq|\lambda| \leq r_{3}^{+}(S)\right\} .
$$

If $r_{2}^{-}(S)>r_{3}^{+}(S)$, then

$$
\sigma_{S}\left(e_{0}\right)=\left\{\lambda \in \mathbb{C}: r_{2}^{-}(S) \leq|\lambda| \leq r_{3}^{+}(S)\right\}=\emptyset .
$$

Thus, we may assume that $r_{2}^{-}(S) \leq r_{3}^{+}(S)$. Recall that the condition $r_{2}^{-}(S)$ $\leq r_{3}^{+}(S)$ means that $S$ has the single-valued extension property (see Proposition 4.1). As at the beginning of the proof of Theorem 3.5, one can show that $\sigma_{S}\left(e_{0}\right)$ has circular symmetry about the origin. Since $r_{S}\left(e_{0}\right)=r_{3}^{+}(S)$, we have

$$
\left\{\lambda \in \mathbb{C}:|\lambda|=r_{3}^{+}(S)\right\} \subset \sigma_{S}\left(e_{0}\right) .
$$

Now, let us show

$$
\left\{\lambda \in \mathbb{C}: r_{2}^{-}(S) \leq|\lambda| \leq r_{3}^{+}(S)\right\} \subset \sigma_{S}\left(e_{0}\right) .
$$


Indeed, from (4.15) we can assume that $r_{2}^{-}(S)<r_{3}^{+}(S)$. By writing $\widetilde{e}_{0}(\lambda)=$ $\sum_{n \in \mathbb{Z}} A_{n}(\lambda) e_{n}, \lambda \in \varrho_{S}\left(e_{0}\right)$, we deduce from the equation

$$
(S-\lambda) \widetilde{e}_{0}(\lambda)=e_{0}, \quad \lambda \in \varrho_{S}\left(e_{0}\right),
$$

that for every $\lambda \in \varrho_{S}\left(e_{0}\right)$ we have

$$
\begin{aligned}
& A_{-1}(\lambda) \omega_{-1}-\lambda A_{0}(\lambda)=1, \\
& A_{n}(\lambda) \omega_{n}-\lambda A_{n+1}(\lambda)=0, \quad n \in \mathbb{Z}, n \neq-1 .
\end{aligned}
$$

From (4.18) we see that for every $\lambda \in \varrho_{S}\left(e_{0}\right)$, we have

$$
\frac{\lambda^{n}}{\beta_{n}} A_{n}(\lambda)=A_{0}(\lambda), \quad A_{-n}(\lambda)=\omega_{-1} \beta_{-n} \lambda^{n-1} A_{-1}(\lambda), \quad n \geq 1 .
$$

Now, suppose that there is $\lambda_{0} \in \varrho_{S}\left(e_{0}\right)$ such that $r_{2}^{-}(S)<\left|\lambda_{0}\right|<r_{3}^{+}(S)$. Then there are two subsequences $\left(n_{k}\right)_{k \geq 0}$ and $\left(m_{k}\right)_{k \geq 0}$ such that for every $k \geq 0$, we have

$$
\left|\lambda_{0}\right|^{n_{k}} \leq \beta_{n_{k}}, \quad 1 / \beta_{-m_{k}} \leq\left|\lambda_{0}\right|^{m_{k}}
$$

Therefore, it follows from (4.19) that for every $k \geq 0$, we have

$$
\left|A_{0}\left(\lambda_{0}\right)\right| \leq\left|A_{n_{k}}\left(\lambda_{0}\right)\right|, \quad\left|A_{-m_{k}}\left(\lambda_{0}\right)\right| \geq \omega_{-1}\left|A_{-1}\left(\lambda_{0}\right)\right| .
$$

Since $\lim _{n \rightarrow \infty} A_{n}\left(\lambda_{0}\right)=\lim _{n \rightarrow \infty} A_{-n}\left(\lambda_{0}\right)=0$, we obtain $A_{0}\left(\lambda_{0}\right)=A_{-1}\left(\lambda_{0}\right)$ $=0$, which contradicts (4.17). Thus, the desired inclusion (4.16) holds.

By combining (4.14) and (4.16) we get

$$
\sigma_{S}\left(e_{0}\right)=\left\{\lambda \in \mathbb{C}: r_{2}^{-}(S) \leq|\lambda| \leq r_{3}^{+}(S)\right\} .
$$

Finally, let $x=\sum_{n \in \mathbb{Z}} a_{n} e_{n} \in \mathcal{H}_{0} \backslash\{0\}$, and let $n_{0}$ be the smallest integer $n$ for which $a_{n} \neq 0$. Then there is a non-zero polynomial $p$ such that $x=$ $p(S) e_{n_{0}}$. If $r_{2}^{-}(S)>r_{3}^{+}(S)$, then the inclusion $\sigma_{S}\left(p(S) e_{n_{0}}\right) \subset \sigma_{S}\left(e_{n_{0}}\right)$ and (4.11) imply that

$$
\sigma_{S}(x)=\left\{\lambda \in \mathbb{C}: r_{2}^{-}(S) \leq|\lambda| \leq r_{3}^{+}(S)\right\}=\emptyset .
$$

So, assume that $r_{2}^{-}(S) \leq r_{3}^{+}(S)$. By Theorem 1.1, we have

$$
\sigma_{S}\left(e_{n_{0}}\right) \backslash Z_{S}\left(p, e_{n_{0}}\right) \subset \sigma_{S}(x)=\sigma_{S}\left(p(S) e_{n_{0}}\right) \subset \sigma_{S}\left(e_{n_{0}}\right) .
$$

Since $Z_{S}\left(p, e_{n_{0}}\right)$ is finite and $\sigma_{S}\left(e_{n_{0}}\right)$ has no isolated points, we see that

$$
\sigma_{S}(x)=\sigma_{S}\left(e_{n_{0}}\right)=\left\{\lambda \in \mathbb{C}: r_{2}^{-}(S) \leq|\lambda| \leq r_{3}^{+}(S)\right\} .
$$

For every non-zero $x=\sum_{n \in \mathbb{Z}} a_{n} e_{n} \in \mathcal{H}$, we set

$$
R_{\omega}^{-}(x):=\limsup _{n \rightarrow \infty}\left|\frac{a_{-n}}{\beta_{-n}}\right|^{1 / n}, \quad R_{\omega}^{+}(x):=\liminf _{n \rightarrow \infty}\left|\frac{\beta_{n}}{a_{n}}\right|^{1 / n} .
$$

Note that $0 \leq R_{\omega}^{-}(x) \leq r_{3}^{-}(S)$ and $r_{2}^{+}(S) \leq R_{\omega}^{+}(x) \leq \infty$ for every non-zero $x \in \mathcal{H}$.

Theorem 4.7. Assume that $r_{2}^{-}(S) \leq r_{3}^{+}(S)$. For every non-zero $x \in \mathcal{H}$, the following statements hold. 
(a) If $R_{\omega}^{-}(x)<r_{2}^{-}(S)$ and $r_{3}^{+}(S)<R_{\omega}^{+}(x)$, then

$$
\sigma_{S}(x)=\left\{\lambda \in \mathbb{C}: r_{2}^{-}(S) \leq|\lambda| \leq r_{3}^{+}(S)\right\} .
$$

(b) Otherwise,

$\left\{\lambda \in \mathbb{C}: \max \left(R_{\omega}^{-}(x), r_{2}^{-}(S)\right)<|\lambda|<\min \left(R_{\omega}^{+}(x), r_{3}^{+}(S)\right)\right\} \subset \sigma_{S}(x)$.

Proof. Recall again that the assumption $r_{2}^{-}(S) \leq r_{3}^{+}(S)$ means that $S$ has the single-valued extension property (see Proposition 4.1). Let $x=$ $\sum_{n \in \mathbb{Z}} a_{n} e_{n} \neq 0$.

(a) Assume that $R_{\omega}^{-}(x)<r_{2}^{-}(S)$ and $r_{3}^{+}(S)<R_{\omega}^{+}(x)$. Keep in mind that $\sigma_{S}\left(e_{0}\right)=\left\{\lambda \in \mathbb{C}: r_{2}^{-}(S) \leq|\lambda| \leq r_{3}^{+}(S)\right\}$ and

$$
\widetilde{e}_{0}(\lambda)= \begin{cases}-\sum_{n=0}^{\infty} \frac{\beta_{n}}{\lambda^{n+1}} e_{n} & \text { if }|\lambda|>r_{3}^{+}(S), \\ \sum_{n=1}^{\infty} \lambda^{n-1} \beta_{-n} e_{-n} & \text { if }|\lambda|<r_{2}^{-}(S) .\end{cases}
$$

Now, consider the analytic $\mathcal{H}$-valued function on the open annulus $\{\lambda \in \mathbb{C}$ : $\left.R_{\omega}^{-}(x)<|\lambda|<R_{\omega}^{+}(x)\right\}$ defined by

$$
f(\lambda):=\sum_{n \in \mathbb{Z}} \frac{a_{n}}{\beta_{n}} \lambda^{n} .
$$

Let $R^{+}$and $R^{-}$be two reals such that

$$
r_{3}^{+}(S)<R^{+}<R_{\omega}^{+}(x), \quad R_{\omega}^{-}(x)<R^{-}<r_{2}^{-}(S) .
$$

Let $\Gamma^{+}$(resp. $\Gamma^{-}$) be the circle centered at the origin and of radius $R^{+}$ (resp. $R^{-}$). Suppose that $\Gamma^{+}$(resp. $\Gamma^{-}$) is anti-clockwise (resp. clockwise) oriented. We have

$$
\begin{aligned}
f\left(S, e_{0}\right)= & \frac{-1}{2 \pi i} \oint_{\lambda \in \Gamma^{+} \cup \Gamma^{-}} f(\lambda) \widetilde{e}_{0}(\lambda) d \lambda=\sum_{n=0}^{\infty}\left[\frac{1}{2 \pi i} \oint_{\lambda \in \Gamma^{+}} \frac{f(\lambda)}{\lambda^{n+1}} d \lambda\right] \beta_{n} e_{n} \\
& +\sum_{n=1}^{\infty}\left[\frac{-1}{2 \pi i} \oint_{\lambda \in \Gamma^{-}} f(\lambda) \lambda^{n-1} d \lambda\right] \beta_{-n} e_{-n}=x .
\end{aligned}
$$

As in the last part of the proof of Proposition 4.6, we conclude from Theorem 1.1 that

$$
\sigma_{S}(x)=\sigma_{S}\left(e_{0}\right)=\left\{\lambda \in \mathbb{C}: r_{2}^{-}(S) \leq|\lambda| \leq r_{3}^{+}(S)\right\} .
$$

(b) Suppose that $\max \left(R_{\omega}^{-}(x), r_{2}^{-}(S)\right)<\min \left(R_{\omega}^{+}(x), r_{3}^{+}(S)\right)$, otherwise there is nothing to prove. By writing $\widetilde{x}(\lambda)=\sum_{n \in \mathbb{Z}} A_{n}(\lambda) e_{n}, \lambda \in \varrho_{S}(x)$, we deduce from the equation

$$
(S-\lambda) \widetilde{x}(\lambda)=x, \quad \lambda \in \varrho_{S}(x),
$$


that for every $\lambda \in \varrho_{S}(x)$, we have

$$
A_{n}(\lambda) \omega_{n}-\lambda A_{n+1}(\lambda)=a_{n+1}, \quad \forall n \in \mathbb{Z} .
$$

In particular, for every $\lambda \in \varrho_{S}(x)$, we have

$$
A_{-1}(\lambda) \omega_{-1}-\lambda A_{0}(\lambda)=a_{0} .
$$

By a simple calculation, we infer from (4.21) that, for every $\lambda \in \varrho_{S}(x)$ and $n \geq 1$,

$$
\frac{\lambda^{n}}{\beta_{n}} A_{n}(\lambda)=A_{0}(\lambda)-\frac{a_{1}}{\beta_{1}}-\frac{a_{2}}{\beta_{2}} \lambda-\ldots-\frac{a_{n}}{\beta_{n}} \lambda^{n-1},
$$

and

$$
A_{-n}(\lambda)=\frac{\lambda^{n-1}}{\omega_{-2} \ldots \omega_{-n}} A_{-1}(\lambda)+\frac{\lambda^{n-2} a_{-1}}{\omega_{-2} \ldots \omega_{-n}}+\ldots+\frac{a_{-n+1}}{\omega_{-n}} .
$$

Note that for every $\lambda \in \varrho_{S}(x), \lambda \neq 0,(4.24)$ can be reformulated as follows:

$$
A_{-n}(\lambda)=\beta_{-n} \lambda^{n-1}\left[\omega_{-1} A_{-1}(\lambda)+\frac{a_{-1}}{\beta_{-1} \lambda}+\frac{a_{-2}}{\beta_{-2} \lambda^{2}}+\ldots+\frac{a_{-n+1}}{\beta_{-n+1} \lambda^{n-1}}\right] .
$$

Now, suppose that

$$
O:=\left\{\lambda \in \mathbb{C}: \max \left(R_{\omega}^{-}(x), r_{2}^{-}(S)\right)<|\lambda|<\min \left(R_{\omega}^{+}(x), r_{3}^{+}(S)\right)\right\} \cap \varrho_{S}(x) \neq \emptyset .
$$

Fix $\lambda \in O$. There are two subsequences $\left(n_{k}\right)_{k \geq 0}$ and $\left(m_{k}\right)_{k \geq 0}$ such that for every $k \geq 0$, we have

$$
|\lambda|^{n_{k}} \leq \beta_{n_{k}}, \quad \frac{1}{\beta_{-m_{k}}} \leq|\lambda|^{m_{k}}
$$

From (4.23) and (4.25), we get, respectively,

and

$$
\left|A_{n_{k}}(\lambda)\right| \geq\left|A_{0}(\lambda)-\frac{a_{1}}{\beta_{1}}-\frac{a_{2}}{\beta_{2}} \lambda-\ldots-\frac{a_{n_{k}}}{\beta_{n_{k}}} \lambda^{n_{k}-1}\right|,
$$

$$
\left|A_{-m_{k}}(\lambda)\right| \geq\left|\omega_{-1} A_{-1}(\lambda)+\frac{a_{-1}}{\beta_{-1} \lambda}+\frac{a_{-2}}{\beta_{-2} \lambda^{2}}+\ldots+\frac{a_{-m_{k}+1}}{\beta_{-m_{k}+1} \lambda^{m_{k}-1}}\right| .
$$

As $\lim _{n \rightarrow \infty} A_{n}(\lambda)=\lim _{n \rightarrow \infty} A_{-n}(\lambda)=0$, and both series $\sum_{n \geq 1}\left(a_{n} / \beta_{n}\right) \lambda^{n-1}$ and $\sum_{n \leq-1}\left(a_{n} / \beta_{n}\right) \lambda^{n}$ converge, it follows that

$$
\begin{aligned}
A_{0}(\lambda) & =\sum_{n=1}^{\infty} \frac{a_{n}}{\beta_{n}} \lambda^{n-1}, \\
\omega_{-1} A_{-1}(\lambda) & =-\sum_{n \leq-1} \frac{a_{n}}{\beta_{n}} \lambda^{n} .
\end{aligned}
$$

From (4.22), (4.26), and (4.27), we see that $\sum_{n \in \mathbb{Z}}\left(a_{n} / \beta_{n}\right) \lambda^{n}=0$ for every $\lambda \in O$; therefore, $a_{n}=0$ for every $n \in \mathbb{Z}$. This contradicts the fact that $x \neq 0$, and completes the proof. 
As a consequence of Theorem 4.7, we deduce the following result. The arguments of the proof are influenced by ideas from [20].

Corollary 4.8. If $T \in \mathcal{L}(\mathcal{H})$ is a non-zero operator that commutes with $S$ then

$$
\left\{\lambda \in \mathbb{C}: r_{2}^{-}(S)<|\lambda|<r_{3}^{+}(S)\right\} \subset \sigma_{S}\left(T e_{n}\right) \subset\left\{\lambda \in \mathbb{C}: r_{2}^{-}(S) \leq|\lambda| \leq r_{3}^{+}(S)\right\}
$$

for every $n \in \mathbb{Z}$.

Proof. By Proposition 4.6, we always have

$$
\sigma_{S}\left(T e_{n}\right) \subset \sigma_{S}\left(e_{n}\right)=\left\{\lambda \in \mathbb{C}: r_{2}^{-}(S) \leq|\lambda| \leq r_{3}^{+}(S)\right\} .
$$

On the other hand, it is easy to verify that $\sigma_{S}\left(T e_{n}\right)=\sigma_{S}\left(T e_{0}\right)$ for all $n \in \mathbb{Z}$. Therefore, it suffices to show that

$$
\left\{\lambda \in \mathbb{C}: r_{2}^{-}(S)<|\lambda|<r_{3}^{+}(S)\right\} \subset \sigma_{S}\left(T e_{0}\right) .
$$

To do this, we shall prove that

$$
R_{\omega}^{-}\left(T e_{0}\right) \leq r_{1}(S), \quad r(S) \leq R_{\omega}^{+}\left(T e_{0}\right) .
$$

This together with Theorem 4.7(b) entails the desired inclusion (4.28). Indeed, write $T e_{0}:=\sum_{n \in \mathbb{Z}} a_{n} e_{n}$ and fix $n \in \mathbb{Z}$. Just as in the proof of Corollary 3.7 , for every $k \geq 0$, we have

$$
\frac{a_{n}}{\beta_{n}} \frac{\beta_{n+k}}{\beta_{k}}=\left\langle T e_{k} ; e_{n+k}\right\rangle .
$$

Similarly, for every $k<0$, we have

$$
\begin{aligned}
a_{n}=\left\langle T e_{0} ; e_{n}\right\rangle & =\beta_{k}\left\langle T S^{-k} e_{k} ; e_{n}\right\rangle=\beta_{k}\left\langle T e_{k} ;\left(S^{*}\right)^{-k} e_{n}\right\rangle \\
& =\frac{\beta_{k} \beta_{n}}{\beta_{n+k}}\left\langle T e_{k} ; e_{n+k}\right\rangle .
\end{aligned}
$$

Therefore, for every $n \geq 0$ and $k \in \mathbb{Z}$, we have

$$
\left|\frac{a_{-n}}{\beta_{-n}}\right| \leq\|T\| \frac{\beta_{k}}{\beta_{k-n}}, \quad \frac{\beta_{n+k}}{\beta_{k}} \leq\|T\|\left|\frac{\beta_{n}}{a_{n}}\right| .
$$

From the right inequality we clearly have $r(S) \leq R_{\omega}^{+}\left(T e_{0}\right)$ whether $S$ is invertible or not. From the left inequality we also clearly have $R_{\omega}^{-}\left(T e_{0}\right) \leq$ $1 / r\left(S^{-1}\right)=r_{1}(S)$ provided that $S$ is invertible. If $S$ is not invertible, then since in this case $\inf _{k \in \mathbb{Z}}\left(\beta_{k} / \beta_{k-n}\right)=0$ for all $n>0$, it follows from the same inequality that $a_{-n}=0$ for all $n>0$ and $R_{\omega}^{-}\left(T e_{0}\right)=0 \leq r_{1}(S)$, as required. Therefore, the inclusion (4.28) is established, and the proof is complete.

Note that Theorem 4.7 is relevant when $r_{2}^{-}(S)>0$. In this case, we computed the local spectrum of $S$ at most points in $\mathcal{H}$. The next result deals with the case $r_{2}^{-}(S)=0$. For every $k \in \mathbb{Z}$, we write

$$
\mathcal{H}_{k}^{+}=\bigvee\left\{e_{n}: n \geq k\right\}, \quad \mathcal{H}_{k}^{-}=\bigvee\left\{e_{n}: n<k\right\}
$$


Theorem 4.9. Assume that $r_{2}^{-}(S)=0$. Let $k \in \mathbb{Z}$. Then for every non-zero $x \in \mathcal{H}_{k}^{+}$, the following statements hold.

(a) If $r_{3}^{+}(S)<R_{\omega}^{+}(x)$, then $\sigma_{S}(x)=\left\{\lambda \in \mathbb{C}:|\lambda| \leq r_{3}^{+}(S)\right\}$.

(b) If $r_{3}^{+}(S) \geq R_{\omega}^{+}(x)$, then $\left\{\lambda \in \mathbb{C}:|\lambda| \leq R_{\omega}^{+}(x)\right\} \subset \sigma_{S}(x)$.

Proof. Since $\mathcal{H}_{k}^{+}$and $\mathcal{H}_{k}^{-}$are invariant subspaces of $S$ and $S^{*}$ respectively, we note that $S^{+}:=S_{\mid \mathcal{H}_{k}^{+}}$and $S^{-}:=S_{\mid \mathcal{H}_{k}^{-}}^{*}$ are unilateral weighted shift operators with respective weight sequences $\omega^{+}:=\left(\omega_{n}\right)_{n \geq k}$ and $\omega^{-}:=$ $\left(\omega_{n}\right)_{n<k}$. We have

$$
r_{i}\left(S^{ \pm}\right)=r_{i}^{ \pm}(S), \quad r\left(S^{ \pm}\right)=r^{ \pm}(S) \quad(i=1,2,3) .
$$

Moreover, for every $x \in \mathcal{H}_{k}^{+}$, we have

$$
R_{\omega}^{+}(x)=R_{\omega^{+}}(x) .
$$

We also note that since $r_{2}\left(S^{-}\right)=r_{2}^{-}(S)=0, S^{-*}$ has the single-valued extension property. As $S$ has a matrix representation

$$
S=\left[\begin{array}{cc}
S^{+} & * \\
0 & S^{-*}
\end{array}\right]
$$

on $\mathcal{H}=\mathcal{H}_{k}^{+} \oplus \mathcal{H}_{k}^{-}$, it follows from Lemma 2.4 that $\sigma_{S}(x)=\sigma_{S^{+}}(x)$ for every $x \in \mathcal{H}_{k}^{+}$. In view of Theorem 3.5, the proof is complete.

4.2. Which bilateral weighted shift operators satisfy Dunford's condition $(C)$ ? The following result gives a necessary condition for a bilateral weighted shift operator $S$ to satisfy Dunford's condition $(C)$.

Theorem 4.10. Assume that $S$ satisfies Dunford's condition $(C)$.

(a) If $S$ is not invertible, then

$$
r_{1}(S)=r_{2}^{-}(S)=0 \leq r_{3}^{+}(S)=r(S) .
$$

(b) If $S$ is invertible, then

$$
r_{1}(S)=1 / r\left(S^{-1}\right)=r_{2}^{-}(S) \leq r_{3}^{+}(S)=r(S) .
$$

Moreover, either the spectrum of $S$ is a circle, or

$$
\sigma_{S}\left(T e_{n}\right)=\sigma(S) \quad(n \in \mathbb{Z})
$$

for every non-zero operator $T \in \mathcal{L}(\mathcal{H})$ that commutes with $S$.

Proof. Note that since $S$ satisfies Dunford's condition $(C), S$ has the single-valued extension property. By Proposition 4.1, we have $r_{2}^{-}(S) \leq$ $r_{3}^{+}(S)$. Now, let $F:=\left\{\lambda \in \mathbb{C}: r_{2}^{-}(S) \leq|\lambda| \leq r_{3}^{+}(S)\right\}$; it follows from Proposition 4.6 that $\mathcal{H}_{S}(F)$ contains a dense subspace. As $\mathcal{H}_{S}(F)$ is closed, we have $\mathcal{H}_{S}(F)=\mathcal{H}$; so, $\sigma_{S}(x) \subset F$ for every $x \in \mathcal{H}$. Since $\sigma(S)=\bigcup_{x \in \mathcal{H}} \sigma_{S}(x) \subset F$ (see Proposition 1.3.2 of [13]) and $F=\sigma_{S}\left(e_{0}\right) \subset \sigma(S)$, we have $\sigma(S)=\{\lambda \in$ $\left.\mathbb{C}: r_{2}^{-}(S) \leq|\lambda| \leq r_{3}^{+}(S)\right\}$, from which assertions (a) and (b) follow.

The remaining assertion is now an immediate consequence of Corollary 4.8 . 
For $T \in \mathcal{L}(\mathcal{H})$, we set

$$
q(T):=\min \{|\lambda|: \lambda \in \sigma(T)\} .
$$

Note that

$$
q(T)= \begin{cases}0 & \text { if } T \text { is not invertible } \\ 1 / r\left(T^{-1}\right) & \text { if } T \text { is invertible. }\end{cases}
$$

REMARK 4.11. An interesting special case occurs when the sequences $\left(\beta_{n}^{1 / n}\right)_{n \geq 1}$ and $\left(\beta_{-n}^{-1 / n}\right)_{n \geq 1}$ converge respectively to $a$ and $b$. By combining Proposition 4.3 and Theorem 4.10, we see that either $a=b$, or the following statements are equivalent.

(a) $S$ has fat local spectra, that is, $\sigma_{S}(x)=\sigma(S)$ for all $x \in \mathcal{H} \backslash\{0\}$.

(b) $S$ satisfies Dunford's condition $(C)$.

(c) $b=q(S) \leq a=r(S)$.

As in the unilateral case, we note that in the general setting if $S$ is not invertible (resp. invertible) and satisfies (4.30) (resp. (4.31)), then $\sigma_{S}(x)=$ $\sigma(S)$ for all non-zero $x$ in a dense subset of $\mathcal{H}$ (see Proposition 4.6, Theorem 4.7 and Theorem 4.9). Thus, we conjecture that if $S$ satisfies Dunford's condition $(C)$ then either $S$ has fat local spectra or its spectrum is a circle.

Note that if the spectrum of a bilateral weighted shift operator $S$ is a circle, then $S$ may have fat local spectra (see Example 4.20); also, $S$ may satisfy Dunford's condition $(C)$ without having fat local spectra. This is the case for example of any non-quasi-nilpotent decomposable bilateral weighted shift operator (see Corollary 4.17). But we do not know in general if a bilateral weighted shift operator $S$ satisfies Dunford's condition $(C)$ whenever its spectrum is a circle.

4.3. Which bilateral weighted shift operators have Bishop's property $(\beta)$ ? Suppose that $S$ has Bishop's property $(\beta)$. Theorem 3.10 of [11] states that:

(a) If $S$ is invertible then $S$ satisfies both (4.32) and (4.33).

(b) If $S$ is not invertible then $S$ satisfies (4.33) and $q(S)=r_{2}^{-}(S)=0$.

On the other hand, it is shown in Theorem 2.7 of [17] that

$$
\begin{aligned}
q(S) & =r_{1}(S)=r_{2}^{-}(S)=r_{3}^{-}(S), \\
r_{2}^{+}(S) & =r_{3}^{+}(S)=r^{+}(S)=r(S) .
\end{aligned}
$$

Therefore, the authors deduce (from Proposition 4.3) that either $S$ has fat local spectra or $r_{1}(S)=r(S)$. Using some ideas from both proofs given in [11] and [17], we refine these results as follows.

THEOREM 4.12. If $S$ has Bishop's property $(\beta)$, then

$$
\begin{aligned}
q(S) & =r_{1}^{-}(S)=r_{2}^{-}(S)=r_{3}^{-}(S)=r^{-}(S), \\
r_{1}^{+}(S) & =r_{2}^{+}(S)=r_{3}^{+}(S)=r^{+}(S)=r(S) .
\end{aligned}
$$


In order to prove this theorem, we shall use the following result from [17].

Proposition 4.13. Assume that $T \in \mathcal{L}(\mathcal{H})$ is injective. If $T$ has Bishop's property $(\beta)$ then for every $x \in T^{\infty} \mathcal{H}$, the sequence $\left(\left\|T^{-n} x\right\|^{-1 / n}\right)_{n \geq 1}$ is convergent and

$$
\lim _{n \rightarrow \infty}\left\|T^{-n} x\right\|^{-1 / n}=\min \left\{|\lambda|: \lambda \in \sigma_{T}(x)\right\} .
$$

Proof of Theorem 4.12. Let $\mathcal{H}_{0}^{+}:=\bigvee\left\{e_{k}: k \geq 0\right\}$ and $S^{+}:=S_{\mid \mathcal{H}_{0}^{+}}$. It is clear that $S^{+}$is a unilateral weighted shift operator with Bishop's property $(\beta)$. Hence, according to Theorem 3.9, we have $r_{1}\left(S^{+}\right)=r\left(S^{+}\right)$. As

$$
r_{i}\left(S^{+}\right)=r_{i}^{+}(S), \quad r\left(S^{+}\right)=r^{+}(S) \quad(i=1,2,3),
$$

and

$$
r_{1}^{+}(S) \leq r_{2}^{+}(S) \leq r_{3}^{+}(S) \leq r^{+}(S)
$$

we have

$$
r_{1}^{+}(S)=r_{2}^{+}(S)=r_{3}^{+}(S)=r^{+}(S) .
$$

Note that since $S$ satisfies Dunford's condition $(C)$, we have $r_{3}^{+}(S)=r(S)$ (see Theorem 4.10). Therefore, from (4.34), we see that

$$
r_{1}^{+}(S)=r_{2}^{+}(S)=r_{3}^{+}(S)=r^{+}(S)=r(S) .
$$

Since $r(S)=\max \left(r^{-}(S), r^{+}(S)\right)$, we have

$$
r^{-}(S) \leq r_{1}^{+}(S)=r_{2}^{+}(S)=r_{3}^{+}(S)=r^{+}(S)=r(S) .
$$

If $S$ is invertible, then $S^{-1}$ also has Bishop's property $(\beta)$ (see Theorem 3.3 .9 of [13]). As

$$
r^{ \pm}\left(S^{-1}\right)=\frac{1}{r_{1}^{\mp}(S)}, \quad r_{1}^{ \pm}\left(S^{-1}\right)=\frac{1}{r^{\mp}(S)}, \quad r_{2,3}^{ \pm}\left(S^{-1}\right)=\frac{1}{r_{3,2}^{\mp}(S)},
$$

the desired conclusion follows by applying (4.35) to $S^{-1}$.

Now, assume that $S$ is not invertible. Since $S$ satisfies Dunford's condition $(C)$, it follows from (4.30) that $r_{1}(S)=r_{2}^{-}(S)=0$. Hence,

$$
q(S)=r_{1}(S)=r_{1}^{-}(S)=r_{2}^{-}(S)=0 .
$$

In view of (4.35) and (4.36), we deduce from Theorem 7 of $[20]$ that

$$
\sigma_{\text {ap }}(S)=\left\{\lambda \in \mathbb{C}:|\lambda| \leq r^{-}(S)\right\} \cup\{\lambda \in \mathbb{C}:|\lambda|=r(S)\} .
$$

Since $S$ is a bi-cyclic operator, it follows from Proposition 2.2 that

$$
\Re\left(S^{*}\right)=\overline{\sigma(S) \backslash \sigma_{\mathrm{ap}}(S)}=\left\{\lambda \in \mathbb{C}: r^{-}(S)<|\lambda|<r(S)\right\} .
$$

On the other hand, we always have

$$
\Re\left(S^{*}\right)=\left\{\lambda \in \mathbb{C}: r_{3}^{-}(S)<|\lambda|<r_{2}^{+}(S)\right\} .
$$


Therefore,

$$
r_{3}^{-}(S)=r^{-}(S) .
$$

Since the sequence $\left(\left\|S^{-n} e_{0}\right\|^{-1 / n}\right)_{n \geq 1}$ is convergent (see Proposition 4.13), we have

$$
r_{2}^{-}(S)=r_{3}^{-}(S) \text {. }
$$

Thus, the desired result follows from (4.36)-(4.38).

Conversely, we have the following result.

Theorem 4.14. Assume that $S$ satisfies (4.32) and (4.33).

(a) If $S$ is not invertible, then either $S$ has Bishop's property $(\beta)$ or $\sigma_{\beta}(S)=\{\lambda \in \mathbb{C}:|\lambda|=r(S)\}$.

(b) If $S$ is invertible, then either $S$ has Bishop's property $(\beta)$, or $\sigma_{\beta}(S)$ equals either one of the circles $\left\{\lambda \in \mathbb{C}:|\lambda|=1 / r\left(S^{-1}\right)\right\}$ and $\{\lambda \in \mathbb{C}:|\lambda|=$ $r(S)\}$, or their union.

Proof. (a) Assume that $S$ is not invertible. If we set $\mathcal{H}_{0}^{+}=\bigvee\left\{e_{k}: k \geq 0\right\}$ and $\mathcal{H}_{0}^{-}=\bigvee\left\{e_{k}: k<0\right\}$, then $S$ has the following matrix representation on $\mathcal{H}=\mathcal{H}_{0}^{+} \oplus \mathcal{H}_{0}^{-}$:

$$
S=\left[\begin{array}{cc}
S^{+} & * \\
0 & S^{-*}
\end{array}\right],
$$

where $S^{+}=S_{\mid \mathcal{H}_{0}^{+}}$and $S^{-}=S_{\mid \mathcal{H}_{0}^{-}}^{*}$ are unilateral weighted shift operators with respective weight sequences $\omega^{+}:=\left(\omega_{n}\right)_{n \geq 0}$ and $\omega^{-}:=\left(\omega_{n}\right)_{n<0}$. Since $r\left(S^{-}\right)=r^{-}(S)=0$ (see (4.32)), $S^{-}$is quasi-nilpotent. In particular, $S^{-*}$ has Bishop's property $(\beta)$. As $r_{1}\left(S^{+}\right)=r_{1}^{+}(S), r\left(S^{+}\right)=r^{+}(S)$ and $r_{1}^{+}(S)=$ $r^{+}(S)=r(S)$ (see (4.33)), we have $r_{1}\left(S^{+}\right)=r\left(S^{+}\right)=r(S)$. By Theorem 3.9 , we see that either $S^{+}$has Bishop's property $(\beta)$ or $\sigma_{\beta}\left(S^{+}\right)=\{\lambda \in \mathbb{C}$ : $\left.|\lambda|=r^{+}(S)\right\}$. Now, the desired conclusion follows from Lemma 2.3.

(b) Assume that $S$ is invertible and satisfies (4.32) and (4.33). It follows from Theorem 7 of [20] that

$$
\sigma_{\text {ap }}(S)=\left\{\lambda \in \mathbb{C}:|\lambda|=1 / r\left(S^{-1}\right)\right\} \cup\{\lambda \in \mathbb{C}:|\lambda|=r(S)\} .
$$

By Proposition 2.1 and Lemma 2.5, the result follows.

REMARK 4.15. As in the unilateral case, we note that if $r_{1}(S)=r(S)$, then either $S$ has Bishop's property $(\beta)$ or $\sigma_{\beta}(S)=\{\lambda \in \mathbb{C}:|\lambda|=r(S)\}$. But if $S$ has Bishop's property $(\beta)$, then $r_{1}(S)$ and $r(S)$ need not be equal as the following example shows. Indeed, let $S$ be the bilateral weighted shift with weights $\left(\omega_{n}\right)_{n \in \mathbb{Z}}$ given by

$$
\omega_{n}= \begin{cases}2 & \text { if } n \geq 0 \\ 1 & \text { if } n<0\end{cases}
$$


It is clear that $S$ is a hyponormal operator; in particular, it has Bishop's property $(\beta)$. On the other hand, we have $r_{1}(S)=1<r(S)=2$.

REMARK 4.16. In view of Remark 4.11 and Theorem 4.12, one can construct examples of bilateral weighted shift operators without Bishop's property $(\beta)$ which satisfy Dunford's condition $(C)$ (see also Example 4.20).

We now give a simple proof of the following well known result.

Corollary 4.17. If $S$ is decomposable then either $S$ is quasi-nilpotent or it is invertible and its spectrum is a circle. In particular, $r_{1}(S)=r(S)$.

Proof. If $S$ is decomposable, then both $S$ and $S^{*}$ have Bishop's property $(\beta)$. Since $S^{*}$ is also a bilateral weighted shift and

$$
r_{i}^{ \pm}\left(S^{*}\right)=r_{i}^{\mp}(S), \quad r^{ \pm}\left(S^{*}\right)=r^{\mp}(S) \quad(i=1,2,3),
$$

the desired result follows by applying Theorem 4.12 to $S$ and $S^{*}$.

The next two propositions give a sufficient condition on the weight $\left(\omega_{n}\right)_{n \in \mathbb{Z}}$ so that the corresponding bilateral weighted shift $S$ is decomposable.

Proposition 4.18. If $m(S)=w(S)$, then $S$ is decomposable.

Proof. Without loss of generality, assume that $m(S)=w(S)=1$. As in the proof of Proposition 3.10, for every $\lambda \notin \sigma(S)=\{\mu \in \mathbb{C}:|\mu|=1\}$ and for every $x \in \mathcal{H}$, we have

$$
|1-| \lambda||\|x\| \leq\|(S-\lambda) x\|,
$$

and Theorem 1.7.2 of [13] shows that $S$ is decomposable.

Proposition 4.19. If $\left(\omega_{n}\right)_{n \in \mathbb{Z}}$ is a periodic sequence, then $S$ is decomposable.

Proof. Suppose that $\left(\omega_{n}\right)_{n \in \mathbb{Z}}$ has period $k$. Then, as in the proof of Proposition 3.12, $\frac{1}{\omega_{0} \ldots \omega_{k-1}} S^{k}$ is an isometry which is clearly invertible. By Proposition 1.6.7 of [13], $S^{k}$ is decomposable, and Theorem 3.3.9 of [13] implies that so is $S$.

Recall that the weight sequence $\left(\omega_{n}\right)_{n \in \mathbb{Z}}$ is said to be almost periodic if there is a periodic positive sequence $\left(p_{n}\right)_{n \in \mathbb{Z}}$ such that $\lim _{|n| \rightarrow \infty}\left(\omega_{n}-p_{n}\right)$ $=0$. Note that if $k$ is the period of $\left(p_{n}\right)_{n \in \mathbb{Z}}$, then

$$
r_{1}(S)=r(S)=\left(p_{0} \ldots p_{k-1}\right)^{1 / k} .
$$

So, one may ask if $S$ is decomposable when the weight $\left(\omega_{n}\right)_{n \in \mathbb{Z}}$ is almost periodic. It turns out that this is not true as the following example shows.

EXAMPLE 4.20. Consider the following real-valued continuous function on $\mathbb{R}$ :

$$
\varphi(x):=\exp \left(\frac{|x| \sin \left[\log \log \log \left(|x|+e^{3}\right)\right]}{\log (|x|+e)}\right)
$$


and set

$$
\omega_{n}=\exp (\varphi(n+1)-\varphi(n)), \quad n \in \mathbb{Z} .
$$

Since $\lim _{|x| \rightarrow \infty} \varphi^{\prime}(x)=0$, we have

$$
\lim _{|n| \rightarrow \infty} \omega_{n}=1 \text {. }
$$

Hence, $\left(\omega_{n}\right)_{n \in \mathbb{Z}}$ is in particular almost periodic and

$$
r_{1}(S)=r(S)=1 \text {. }
$$

On the other hand, it is shown in [6] that $\left(\omega_{n}\right)_{n \in \mathbb{Z}}$ satisfies all the hypotheses of Theorem 2 of [6]. It follows from the proof of that theorem that

$$
\sigma_{S}(x)=\sigma_{S^{*}}(x)=\sigma(S)=\{\lambda \in \mathbb{C}:|\lambda|=1\} \quad \text { for every non-zero } x \in \mathcal{H} \text {. }
$$

This shows that both $S$ and $S^{*}$ have fat local spectra, and so satisfy Dunford's condition $(C)$, but neither $S$ nor $S^{*}$ has Bishop's property $(\beta)$.

5. Examples and comments. The quasi-nilpotent part of an operator $T \in \mathcal{L}(\mathcal{H})$ is the set

$$
\mathcal{H}_{0}(T)=\left\{x \in \mathcal{H}: \lim _{n \rightarrow \infty}\left\|T^{n} x\right\|^{1 / n}=0\right\} .
$$

It is a linear subspace of $\mathcal{H}$, generally not closed. The operator $T$ is said to have property $(Q)$ if $\mathcal{H}_{0}(T-\lambda)$ is closed for every $\lambda \in \mathbb{C}$. Note that Dunford's condition $(C)$ implies property $(Q)$ and in its turn property $(Q)$ implies the single-valued extension property. In [1], the authors have shown, by examples of a convolution operator on group algebras and a direct sum of unilateral weighted shits, that the above implications cannot be reversed in general. The purpose of this section is to provide elementary results in order to produce simpler counter-examples showing that property $(Q)$ is strictly intermediate between Dunford's condition $(C)$ and the single-valued extension property.

Throughout the remainder of this paper, we suppose that $S$ is a unilateral weighted shift operator on $\mathcal{H}$ and the weights $\omega_{n}(n \geq 0)$ are non-negative. Note that $S$ is injective if and only if none of the weights is zero.

It is shown in Proposition 17 of [2] that if $S$ is an injective unilateral weighted shift operator, then $\mathcal{H}_{0}(S)+\operatorname{ran}(S)$ is dense in $\mathcal{H}$ if and only if $r_{3}(S)=0$. However, in view of (3.1) and the fact that $r_{S}\left(e_{n}\right)=r_{3}(S)$ for all $n \geq 0$, one can see that either $\mathcal{H}_{0}(S)=\{0\}$ or $\mathcal{H}_{0}(S)$ is dense in $\mathcal{H}$. More precisely, we have

Proposition 5.1. If $S$ is an injective unilateral weighted shift operator, then $\mathcal{H}_{0}(S)$ is dense in $\mathcal{H}$ if and only if $r_{3}(S)=0$.

The following two propositions enable us to produce examples of operators which have the single-valued extension property without having prop$\operatorname{erty}(Q)$. 
Proposition 5.2. The following assertions are equivalent.

(a) $S^{*}$ is quasi-nilpotent.

(b) $S^{*}$ is decomposable.

(c) $S^{*}$ has Bishop's property $(\beta)$.

(d) $S^{*}$ satisfies Dunford's condition $(C)$.

(e) $S^{*}$ has property $(Q)$.

(f) $\mathcal{H}_{0}\left(S^{*}\right)$ is closed.

Proof. It suffices to prove the implication (f) $\Rightarrow($ a). For every $n \geq 0$, we have $S^{* n+1} e_{n}=0$. This shows that $e_{n} \in \mathcal{H}_{0}\left(S^{*}\right)$ for every $n \geq 0$. Since $\mathcal{H}_{0}\left(S^{*}\right)$ is closed, we have $\mathcal{H}_{0}\left(S^{*}\right)=\mathcal{H}$, and it follows from Theorem 1.5 of $[22]$ that $S^{*}$ is quasi-nilpotent.

A similar proof yields the following result.

Proposition 5.3. If infinitely many weights $\omega_{n}$ are zero, then the following statements are equivalent.

(a) $S$ is quasi-nilpotent.

(b) $S$ is decomposable.

(c) $S$ has Bishop's property $(\beta)$.

(d) S satisfies Dunford's condition $(C)$.

(e) $S$ has property $(Q)$.

(f) $\mathcal{H}_{0}(S)$ is closed.

ExAMPLE 5.4. Note that if $S$ is injective, then $S^{*}$ has the single-valued extension property if and only if $r_{2}(S)=0$. Thus, in view of Proposition 5.2 , we see that if $0=r_{2}(S)<r(S)$, then $S^{*}$ has the single-valued extension property but not property $(Q)$.

It remains to produce a positive bounded weight sequence $\left(\omega_{n}\right)_{n \geq 0}$ such that the corresponding weighted shift operator $S$ satisfies $0=r_{2}(S)<r(S)$. Let $\left(k_{i}\right)_{i \geq 1}$ be the sequence given by

$$
k_{1}=1, \quad k_{i+1}=(i+1) k_{i}+1, \quad i \geq 1 .
$$

Let $\left(\omega_{n}\right)_{n \geq 0}$ be the weight sequence given by

$$
\omega_{n}= \begin{cases}1 / 2^{i k_{i}} & \text { if } n=k_{i}-1 \text { for some } i \geq 1 \\ 2 & \text { otherwise. }\end{cases}
$$

It is easy to check that $\beta_{n} \leq 1$ for every $n \geq 0$, and that

$$
\beta_{k_{i}-1}=1, \quad \beta_{k_{i}}=\frac{1}{2^{i k_{i}}}, \quad i \geq 1 \text {. }
$$

This shows that $0=r_{2}(S)<1=r_{3}(S) \leq r(S)$. Therefore, $S^{*}$ has the single-valued extension property but fails property $(Q)$. 
EXAMPLE 5.5. Let $\left(\omega_{n}\right)_{n \geq 0}$ be the weight sequence given by

$$
\omega_{n}= \begin{cases}0 & \text { if } n \text { is the square of an integer, } \\ 1 & \text { otherwise. }\end{cases}
$$

It is easy to see that $\left\|S^{n}\right\|=1$ for every $n \geq 1$. This shows that $S$ is not quasi-nilpotent, and so does not have property $(Q)$ (see Proposition 5.3). On the other hand, $S$ has the single-valued extension property since $\sigma_{\mathrm{p}}(S)=\{0\}$.

If infinitely many weights $\omega_{n}$ are zero, then by Proposition $5.3, S$ enjoys property $(Q)$ if and only if it is quasi-nilpotent. If only finitely many weights $\omega_{n}$ are zero, then $S$ is the direct sum of a finite-dimensional nilpotent operator $S_{1}$ and an injective unilateral weighted shift $S_{2}$. Therefore, $S$ has property $(Q)$ if and only if $S_{2}$ does. Thus when studying which unilateral weighted shift operator enjoy property $(Q)$ we may assume that none of the weights $\omega_{n}$ is zero.

Proposition 5.6. If $S$ is injective, then the following statements are equivalent.

(a) $S$ has property $(Q)$.

(b) Either $S$ is quasi-nilpotent or $r_{3}(S)>0$.

Proof. Since $S$ has the single-valued extension property, it follows that $\mathcal{H}_{0}(S-\lambda)=\mathcal{H}_{S}(\{\lambda\})$ for every $\lambda \in \mathbb{C}$.

Assume that $r_{3}(S)>0$. For every non-zero $x \in \mathcal{H}, \sigma_{S}(x)$ is a connected set containing 0 (see Corollary 3.2) and $r_{3}(S) \leq r_{S}(x) \leq r(S)$ (see (3.1)). This implies that $\mathcal{H}_{0}(S-\lambda)=\mathcal{H}_{S}(\{\lambda\})=\{0\}$ for every $\lambda \in \mathbb{C}$. Therefore, $S$ has property $(Q)$ and the implication $(\mathrm{b}) \Rightarrow(\mathrm{a})$ holds.

Now, suppose that $S$ has property $(Q)$ and $r_{3}(S)=0$. From Proposition 5.1 we see that $\mathcal{H}_{0}(S)$ is a dense subspace of $\mathcal{H}$. As $\mathcal{H}_{0}(S)$ is closed, we have $\mathcal{H}_{0}(S)=\mathcal{H}$. By Theorem 1.5 of [22], $S$ is quasi-nilpotent. This finishes the proof.

To separate Dunford's condition $(C)$ and property $(Q)$ we need to produce a positive bounded weight sequence $\left(\omega_{n}\right)_{n \geq 0}$ such that the corresponding weighted shift operator $S$ satisfies $0<r_{3}(\bar{S})<r(S)$ (see Theorem 3.8 and Proposition 5.6).

EXAMPLE 5.7. Let $\left(C_{k}\right)_{k \geq 0}$ be a sequence of successive disjoint segments covering the set $\mathbb{N}$ of non-negative integers such that each $C_{k}$ contains $k^{2}$ elements. Let $k \in \mathbb{N}$ and for $n \in C_{k}$ set

$$
\omega_{n}= \begin{cases}2 & \text { if } n \text { is one of the first } k \text { terms of } C_{k}, \\ 1 & \text { otherwise. }\end{cases}
$$

It is easy to see that $r_{1}(S)=r_{3}(S)=1<r(S)=2$. Therefore, $S$ has property $(Q)$ but does not satisfy Dunford's condition $(C)$. 
The idea of this construction is due to W. C. Ridge [19].

Finally, we would like to point out that:

(a) Proposition 5.1 remains valid for an injective bilateral weighted shift operator $S$ upon replacing $r_{3}(S)$ with $r_{3}^{+}(S)$.

(b) If $S$ is a non-injective bilateral weighted shift operator then $S$ is a direct sum of a unilateral weighted shift operator and a backward weighted shift operator. Therefore, the local spectral properties of $S$ can be deduced from the preceding results.

(c) All the results of the present paper remain valid for the weighted shift operators on $l^{p}$-Banach spaces.

Added in proof. After the first version of this paper, [11], had been submitted for publication, the author learned from Professor M. M. Neumann that some results of [11] had also appeared in [17]. In [17], the authors also established some results absent in the present paper about inner and outer radii of arbitrary Banach space operators and gave a growth condition for a Banach space operator to have Bishop's property $(\beta)$. The author expresses his gratitude to Professor M. M. Neumann.

He also thanks Professor P. Aiena and the referee for useful comments.

\section{References}

[1] P. Aiena, M. L. Colasante and M. González, Operators which have a closed quasinilpotent part, Proc. Amer. Math. Soc. 130 (2002), 2701-2710.

[2] P. Aiena, T. L. Miller and M. M. Neumann, On a localized single-valued extension property, Proc. Roy. Irish Acad., to appear.

[3] E. Albrecht and J. Eschmeier, Analytic functional models and local spectral theory, Proc. London Math. Soc. (3) 75 (1997), 323-348.

[4] C. Apostol, Spectral decompositions and functional calculus, Rev. Roumaine Math. Pures Appl. 13 (1968), 1481-1528.

[5] A. Atzmon, Power regular operators, Trans. Amer. Math. Soc. 347 (1995), 31013109 .

[6] A. Atzmon and M. Sodin, Completely indecomposable operators and a uniqueness theorem of Cartwright-Levinson type, J. Funct. Anal. 169 (1999), 164-188; addendum, ibid. 175 (2000), 248-249.

[7] C. A. Berger and J. G. Stampfli, Mapping theorems for the numerical range, Amer. J. Math. 89 (1967) 1047-1055.

[8] T. Bermúdez, M. González and A. Martinón, Properties of the local functional calculus, Proc. Roy. Irish Acad. 102A (2002), 215-225.

[9] —, —, - Stability of the local spectrum, Proc. Amer. Math. Soc. 125 (1997) 417425 .

[10] A. Bourhim, On the largest analytic set for cyclic operators, Int. J. Math. Math. Sci. 30 (2003), 1899-1909.

[11] —, On the local spectral properties of weighted shift operators, The Abdus Salam ICTP Preprint, 2002. 
[12] I. Colojoară and C. Foiaş, Theory of Generalized Spectral Operators, Gordon and Breach, New York, 1968.

[13] K. B. Laursen and M. M. Neumann, An Introduction to Local Spectral Theory, London Math. Soc. Monogr. 20, Clarendon Press, 2000.

[14] E. Makai and J. Zemánek, The surjectivity radius, packing numbers and boundedness below of linear operators, Integral Equations Operator Theory 6 (1983), 372-384.

[15] P. McGuire, A local functional calculus, ibid. 9 (1986), 218-236.

[16] T. L. Miller and V. G. Miller, An operator satisfying Dunford's condition $(C)$ but without Bishop's property ( $\beta$ ), Glasgow Math. J. 40 (1998), 427-430.

[17] T. L. Miller, V. G. Miller and M. M. Neumann, Local spectral properties of weighted shifts, J. Operator Theory, to appear.

[18] M. Radjabalipour, Decomposable operators, Bull. Iranian Math. Soc. 9 (1978), 1L49L.

[19] W. C. Ridge, Approximate point spectrum of a weighted shift, Trans. Amer. Math. Soc. 147 (1970), 349-356.

[20] A. L. Shields, Weighted shift operators and analytic function theory, in: Topics in Operator Theory, C. Pearcy (ed.), Math. Surveys 13, Amer. Math. Soc., Providence, RI, 1974, 49-128.

[21] T. Tam, On a conjecture of Ridge, Proc. Amer. Math. Soc. 125 (1997), 3581-3592.

[22] P. Vrbová, On local spectral properties of operators in Banach spaces, Czechoslovak Math. J. 23 (1973), 483-492.

[23] L. R. Williams, A local functional calculus and related results on the single-valued extension property, Integral Equations Operator Theory 45 (2003), 485-502.

[24] L. Yang, Hyponormal and subdecomposable operators, J. Funct. Anal. 112 (1993), 204-217.

Abdus Salam ICTP

Mathematics Section

11 Miramare

34100 Trieste, Italy

E-mail: bourhim@ictp.trieste.it
Current address: Département de Mathématiques et de Statistique Université Laval, Québec

Canada, G1K 7P4

E-mail: bourhim@mat.ulaval.ca

Received August 2, 2002

Revised version November 5, 2003 